\title{
Interplay effects on blow-up of weakly coupled systems for semilinear wave equations with general nonlinear memory terms
}

\author{
Wenhui Chen*1 \\ ${ }^{1}$ Institute of Applied Analysis, Faculty of Mathematics and Computer Science, Technical \\ University Bergakademie Freiberg, Prüferstraße 9, 09596 Freiberg, Germany
}

\begin{abstract}
In this paper, we study weakly coupled systems for semilinear wave equations with distinct nonlinear memory terms in general forms, and the corresponding single semilinear equation with general nonlinear memory terms. Thanks to Banach's fixed point theorem, we prove local (in time) existence of solutions with the $L^{1}$ assumption on the memory kernels. Then, blow-up results for energy solutions are derived applying iteration methods associated with slicing procedure. We investigate interactions on the blow-up conditions under different decreasing assumptions on the memory term. Particularly, a new threshold for the kernels on interplay effect is found. Additionally, we give some applications of our results on semilinear wave equations and acoustic wave equations.
\end{abstract}

Keywords: Weakly coupled system, semilinear wave equation, blow-up, nonlinear memory term, iteration method.

AMS Classification (2010) Primary: 35L71, 35B44; Secondary: 35L05, 35B33.

\section{Introduction}

In this paper, we mainly work on the Cauchy problem of weakly coupled systems for semilinear wave equations with distinct nonlinear memory terms in general ways, namely,

$$
\begin{cases}u_{t t}-\Delta u=g_{1} *|v|^{p}, & x \in \mathbb{R}^{n}, t>0 \\ v_{t t}-\Delta v=g_{2} *|u|^{q}, & x \in \mathbb{R}^{n}, t>0 \\ \left(u, u_{t}, v, v_{t}\right)(0, x)=\left(u_{0}, u_{1}, v_{0}, v_{1}\right)(x), & x \in \mathbb{R}^{n}\end{cases}
$$

where $p, q>1$, and the convolution nonlinear terms with respect to time variable are denoted by

$$
\left(g_{k} *|w|^{r}\right)(t, x):=\int_{0}^{t} g_{k}(t-\tau)|w(\tau, x)|^{r} \mathrm{~d} \tau
$$

with the time-dependent memory kernels or the so-called relaxation functions $g_{k}=g_{k}(t)>0$ for $k=1,2$. In the above notation of the convolution, we denote $w=u, v$ and $r=p, q$. Our main attempt of the paper is to investigate blow-up conditions for the weakly coupled systems (1.1) influenced by various assumptions on the memory kernels. Moreover, as the special case of (1.1), blow-up results for the corresponding single semilinear wave equation with memory nonlinearities to (1.1) also will be shown.

\footnotetext{
${ }^{*}$ Corresponding author: Wenhui Chen (wenhui.chen.math@gmail.com)
} 
Let us present a historical overview on some results for semilinear wave equations, which are strongly linked with our model and the motivation of considering the weakly coupled systems (1.1). In the last forty years, the following Cauchy problem for semilinear wave equations:

$$
\begin{cases}u_{t t}-\Delta u=f(u ; p), & x \in \mathbb{R}^{n}, t>0, \\ \left(u, u_{t}\right)(0, x)=\left(u_{0}, u_{1}\right)(x), & x \in \mathbb{R}^{n}\end{cases}
$$

with $p>1$ has caught a lot of attention. Concerning the semilinear wave equation with the power nonlinearity, i.e $(1.2)$ with $f(u ; p)=|u|^{p}$, the critical exponent is the so-called Strauss exponent $p_{\mathrm{Str}}(n)$, which is the positive root of the quadratic equation $(n-1) p_{\mathrm{Str}}^{2}-(n+1) p_{\mathrm{Str}}-2=0$ for $n \geqslant 2$, to be specific, it can be represented explicitly by

$$
p_{\text {Str }}(n):=\frac{n+1+\sqrt{n^{2}+10 n-7}}{2(n-1)} \text { for } n \geqslant 2,
$$

and in the one spatial dimensional case $p_{\text {Str }}(1):=\infty$. Note that the critical exponent describes the threshold between global (in time) existence of small data weak solutions and blow-up of local (in time) small data weak solutions. For the finding of the Strauss' conjecture and its proof on the critical exponent, we refer to the classical works $[18,19,32,31,30,15,16,35,26,13,33,17,34,36]$. The previous results, expressly, proved blow-up of weak solutions provided $1<p \leqslant p_{\text {Str }}(n)$. Let us now turn to the study of wave equations with nonlinear memory terms. Recently, the authors of [2] considered the Riemann-Liouville fractional integral of $1-\gamma$ of the $p$ power of solution as the nonlinear terms on the semilinear wave equation (1.2), exactly,

$$
f(u ; p)=\frac{1}{\Gamma(1-\gamma)} \int_{0}^{t}(t-\tau)^{-\gamma}|u(\tau, x)|^{p} \mathrm{~d} \tau \quad \text { with } \quad \gamma \in(0,1)
$$

where $\Gamma$ stands for the Euler integral of the second kind. They investigated a generalized Strauss exponent $p_{0}(n, \gamma)$ defined by

$$
p_{0}(n, \gamma):=\frac{n+3-2 \gamma+\sqrt{n^{2}+(14-4 \gamma) n+(3-2 \gamma)^{2}-8}}{2(n-1)} \text { for } n \geqslant 2 \text {, }
$$

and in the one spatial dimensional case $p_{0}(1, \gamma):=\infty$. Actually, $p_{0}(n, \gamma)$ is the positive root of the quadratic equation $(n-1) p_{0}^{2}-(n+3-2 \gamma) p_{0}-2=0$. The authors of [2] proved blow-up of energy solutions to the semilinear wave equation (1.2) with nonlinearities (1.3) if $1<p \leqslant p_{0}(n, \gamma)$ for $n \geqslant 2$, and $p>1$ for $n=1$, which satisfy $\lim _{\gamma \rightarrow 1^{-}} p_{0}(n, \gamma)=p_{\text {Str }}(n)$ for all $n \geqslant 1$. For this reason, it seems reasonable to consider the general nonlinear memory terms $f(u ; p)=g *|u|^{p}$ by taking suitable assumptions on the memory kernel $g=g(t)$. However, not only global (in time) existence but also blow-up results are still unknown. It seems interesting to find the influence of the memory kernel on these results. We will answer this question in Corollary 2.1 by considering the special case of (1.1) from the point of view of blow-up for energy solutions. Concerning other studies of hyperbolic equations with nonlinear memory terms (1.3), we refer the interested readers to $[12,4,5,23,6,7]$.

On the other hand, it is known that the critical curve in the $p-q$ plane for the Cauchy problem 
for weakly coupled systems of semilinear wave equations

$$
\begin{cases}u_{t t}-\Delta u=|v|^{p}, & x \in \mathbb{R}^{n}, t>0 \\ v_{t t}-\Delta v=|u|^{q}, & x \in \mathbb{R}^{n}, t>0 \\ \left(u, u_{t}, v, v_{t}\right)(0, x)=\left(u_{0}, u_{1}, v_{0}, v_{1}\right)(x), & x \in \mathbb{R}^{n}\end{cases}
$$

is given by the cubic relation

$$
\alpha_{\mathrm{W}}(p, q):=\max \left\{\frac{p+2+q^{-1}}{p q-1}, \frac{q+2+p^{-1}}{p q-1}\right\}=\frac{n-1}{2} .
$$

Concerning the work on this critical curve, we refer to [10, 8, 9, 1, 21, 20, 14, 29, 22]. Particularly, every local (in time) weak solution to the weakly coupled system (1.4) blows up if $\alpha_{\mathrm{W}}(p, q) \geqslant$ $(n-1) / 2$. We should remark that the research of semilinear weakly coupled systems is not just a trivial generalization of those for single semilinear equations, specifically in the case when $p \neq q$. More detail expansions on this effect were pointed out in the recent paper [27].

The main goal of this paper is to investigate blow-up results for the weakly hyperbolic coupled systems (1.1) with distinct and general memory kernels. Specifically, we are interested in the interplay effects between $g_{1}(t)$ and $g_{2}(t)$ on the blow-up conditions.

Roughly speaking, as we will show in Section 2, when the memory kernel decreases slower than $t^{-1}$ (slow decrease), we may feel the influence from the memory kernel on the condition for blow-up of energy solutions. Nonetheless, this phenomenon will disappear when the memory kernel decreases faster than $t^{-1}$ (fast decrease), and in this case the blow-up condition for the exponents $p, q$ is the same as those for the weakly coupled systems (1.4). Therefore, one of our novelties is to derive a new threshold $t^{-1}$ for the long time behavior of memory kernels in the weakly coupled systems (1.1) by using iteration methods. Additionally, in the case when both memory kernels decrease slower than $t^{-1}$, up to the author best knowledge, the blow-up condition with combined effects from $g_{1}(t)$ and $g_{2}(t)$ is attractive. We will give some applications of the obtained results which are the wave equations with the Riemann-Liouville fractional integral type nonlinear memory terms as the example for slow decay memory kernels, and the semilinear Moore-Gibson-Thompson equations in the conservative case as the instance for fast decay memory kernels.

Lastly, we should mention that the study of blow-up for (1.1) is not a simple generalization of those in the previous researches on semilinear wave equations with power nonlinearities. For one thing, due to the memory terms on the nonlinearities, it seems that the classical coupled Kato's type lemma in [10] does not work well in our model. For another, we now do not take any explicit forms of nonlinear memory terms, which give us some technical difficulties in the treatments. To overcome these difficulties, in the present paper, we will employ iteration methods, especially, when the memory kernel decreases fast, slicing procedure (see, for example, [1, 3]) will be used.

The remaining part of the paper is organized as follows. We will show our main results on local (in time) existence and blow-up, moreover, give some applications on these results in Section 2. We next prove existence of local (in time) solution by applying Banach's fixed point theorem and $L^{2}-L^{2}$ estimates in Section 3. Then, the proof of the blow-up results by using iteration methods will be shown in Section 4. Eventually, final remarks concerning some open problems in Section 5 complete the present paper. 
Notation: We give some notations to be used in this paper. We write $f \lesssim g$ when there exists a positive constant $C$ such that $f \leqslant C g$ and, similarly, for $f \gtrsim g$. The relation $f \asymp g$ means that $f \lesssim g \lesssim f$. We denote $\lceil r\rceil:=\min \{C \in \mathbb{Z}: r \leqslant C\}$ as the ceiling function. Moreover, $B_{R}$ denotes the ball around the origin with radius $R$ in $\mathbb{R}^{n}$.

\section{Main results}

Let us first state the result for local (in time) existence of solutions of the weakly coupled systems (1.1) under a assumption on the memory kernels.

Theorem 2.1. Let $\left(u_{0}, u_{1}, v_{0}, v_{1}\right) \in\left(H^{1}\left(\mathbb{R}^{n}\right) \times L^{2}\left(\mathbb{R}^{n}\right)\right) \times\left(H^{1}\left(\mathbb{R}^{n}\right) \times L^{2}\left(\mathbb{R}^{n}\right)\right)$ compactly support in a ball with some radials $R>0$. We assume $p, q>1$ such that $p, q \leqslant n /(n-2)$ when $n \geqslant 3$. Then, there exists a positive $T$ and a uniquely determined local (in time) mild solution

$$
(u, v) \in\left(\mathscr{C}\left([0, T], H^{1}\left(\mathbb{R}^{n}\right)\right) \cap \mathscr{C}^{1}\left([0, T], L^{2}\left(\mathbb{R}^{n}\right)\right)\right)^{2}
$$

to the weakly coupled systems (1.1) with $g_{1}(t), g_{2}(t) \in L^{1}([0, T])$ satisfying $\operatorname{supp} u(t, \cdot)$, supp $v(t, \cdot) \subset$ $B_{R+t}$ for any $t \in[0, T]$.

Before stating our main results for blow-up of solutions, motivated by [25] we first define a suitable energy solutions of the weakly coupled systems (1.1).

Definition 2.1. Let $\left(u_{0}, u_{1}, v_{0}, v_{1}\right) \in\left(H^{1}\left(\mathbb{R}^{n}\right) \times L^{2}\left(\mathbb{R}^{n}\right)\right) \times\left(H^{1}\left(\mathbb{R}^{n}\right) \times L^{2}\left(\mathbb{R}^{n}\right)\right)$. We say that $(u, v)$ is an energy solution of the hyperbolic coupled systems (1.1) on $[0, T)$ if

$$
\begin{aligned}
& u \in \mathscr{C}\left([0, T), H^{1}\left(\mathbb{R}^{n}\right)\right) \cap \mathscr{C}^{1}\left([0, T), L^{2}\left(\mathbb{R}^{n}\right)\right) \text { with } g_{2} *|u|^{q} \in L_{\mathrm{loc}}^{1}\left([0, T) \times \mathbb{R}^{n}\right), \\
& v \in \mathscr{C}\left([0, T), H^{1}\left(\mathbb{R}^{n}\right)\right) \cap \mathscr{C}^{1}\left([0, T), L^{2}\left(\mathbb{R}^{n}\right)\right) \text { with } g_{1} *|v|^{p} \in L_{\mathrm{loc}}^{1}\left([0, T) \times \mathbb{R}^{n}\right) \text {, }
\end{aligned}
$$

fulfills $(u, v)(0, \cdot)=\left(u_{0}, v_{0}\right)$ in $H^{1}\left(\mathbb{R}^{n}\right) \times H^{1}\left(\mathbb{R}^{n}\right)$ and the integral relations

$$
\begin{aligned}
& \int_{0}^{t} \int_{\mathbb{R}^{n}}\left(-u_{t}(s, x) \phi_{s}(s, x)+\nabla u(s, x) \cdot \nabla \phi(s, x)\right) \mathrm{d} x \mathrm{~d} s+\int_{\mathbb{R}^{n}} u_{t}(t, x) \phi(t, x) \mathrm{d} x \\
& =\int_{\mathbb{R}^{n}} u_{1}(x) \phi(0, x) \mathrm{d} x+\int_{0}^{t} \int_{\mathbb{R}^{n}}\left(g_{1} *|v|^{p}\right)(s, x) \phi(s, x) \mathrm{d} x \mathrm{~d} s
\end{aligned}
$$

as well as

$$
\begin{aligned}
& \int_{0}^{t} \int_{\mathbb{R}^{n}}\left(-v_{t}(s, x) \psi_{s}(s, x)+\nabla v(s, x) \cdot \nabla \psi(s, x)\right) \mathrm{d} x \mathrm{~d} s+\int_{\mathbb{R}^{n}} v_{t}(t, x) \psi(t, x) \mathrm{d} x \\
& =\int_{\mathbb{R}^{n}} v_{1}(x) \psi(0, x) \mathrm{d} x+\int_{0}^{t} \int_{\mathbb{R}^{n}}\left(g_{2} *|u|^{q}\right)(s, x) \psi(s, x) \mathrm{d} x \mathrm{~d} s
\end{aligned}
$$

for any test functions $\phi, \psi \in \mathscr{C}_{0}^{\infty}\left([0, T) \times \mathbb{R}^{n}\right)$ and any $t \in(0, T)$.

By applying once integration by parts in (2.1) and (2.2), one may immediately observe the resultant equalities

$$
\begin{aligned}
& \int_{0}^{t} \int_{\mathbb{R}^{n}} u(s, x)\left(\phi_{s s}(s, x)-\Delta \phi(s, x)\right) \mathrm{d} x \mathrm{~d} s+\int_{\mathbb{R}^{n}}\left(u_{t}(t, x) \phi(t, x)-u(t, x) \phi_{s}(t, x)\right) \mathrm{d} x \\
& =\int_{\mathbb{R}^{n}}\left(u_{1}(x) \phi(0, x)-u_{0}(x) \phi_{s}(0, x)\right) \mathrm{d} x+\int_{0}^{t} \int_{\mathbb{R}^{n}}\left(g_{1} *|v|^{p}\right)(s, x) \phi(s, x) \mathrm{d} x \mathrm{~d} s
\end{aligned}
$$


as well as

$$
\begin{aligned}
& \int_{0}^{t} \int_{\mathbb{R}^{n}} v(s, x)\left(\psi_{s s}(s, x)-\Delta \psi(s, x)\right) \mathrm{d} x \mathrm{~d} s+\int_{\mathbb{R}^{n}}\left(v_{t}(t, x) \psi(t, x)-v(t, x) \psi_{s}(t, x)\right) \mathrm{d} x \\
& =\int_{\mathbb{R}^{n}}\left(v_{1}(x) \psi(0, x)-v_{0}(x) \psi_{s}(0, x)\right) \mathrm{d} x+\int_{0}^{t} \int_{\mathbb{R}^{n}}\left(g_{2} *|u|^{q}\right)(s, x) \psi(s, x) \mathrm{d} x \mathrm{~d} s
\end{aligned}
$$

satisfying the definition of weak solutions of the hyperbolic system (1.1) by letting $t \rightarrow T$.

To describe the interplay effects of $g_{1}(t)$ and $g_{2}(t)$, we now introduce a monotonously increasing function by

$$
\mathscr{L}(t):=\underbrace{\ln (\ln (\cdots \ln }_{r+1 \text { times } \ln } \underbrace{\exp (\exp (\cdots \exp }_{r \text { times } \exp }(1)))+t)))
$$

for any $r \in \mathbb{N}_{0}$ and $t \geqslant 0$. It carries the initial value for $t=0$ such that $\mathscr{L}(0)=0$. The aim of constructing $\mathscr{L}(t)$ is to find a nonnegative function goes to $\infty$ as $t \rightarrow \infty$ with the slower increasing rate. Later, we may choose arbitrary nonnegative integer $r$ in all results, even sufficiently large $r$.

Theorem 2.2. Let $p, q>1$ such that $p, q \leqslant n /(n-2)$ when $n \geqslant 3$. Let us consider $g_{k}(t) \in L^{1}([0, T])$ for $k=1,2$ such that one of the following condition holds:

- if $g_{k}(t) \gtrsim t^{-1}$ for any $t \geqslant t_{0}$ with $t_{0} \in[0, T)$ and $g_{k}^{\prime}(t) \leqslant 0$ for $k=1,2$, then we consider $(p, q)$ so that the next estimate is satisfied:

$$
g_{1}(t) g_{2}(t) \max \left\{\left(g_{1}(t)\right)^{q-1} t^{2 q+1 / p},\left(g_{2}(t)\right)^{p-1} t^{2 p+1 / q}\right\} \gtrsim t^{\frac{n-1}{2}(p q-1)-3} \mathscr{L}(t)
$$

- if $g_{k}(t) \lesssim t^{-1}$ for any $t \geqslant t_{0}$ with $t_{0} \in[0, T)$ and $g_{k}(t) \in \mathscr{C}^{2}([0, T])$ with $g_{k}^{\prime \prime}(0)>0$ for $k=1,2$, then we consider $(p, q)$ so that the next estimate is satisfied:

$$
\max \left\{\frac{p+2+q^{-1}}{p q-1}, \frac{q+2+p^{-1}}{p q-1}\right\}>\frac{n-1}{2} .
$$

Let us suppose that $\left(u_{0}, u_{1}, v_{0}, v_{1}\right) \in\left(H^{1}\left(\mathbb{R}^{n}\right) \times L^{2}\left(\mathbb{R}^{n}\right)\right) \times\left(H^{1}\left(\mathbb{R}^{n}\right) \times L^{2}\left(\mathbb{R}^{n}\right)\right)$ are nonnegative and compactly supported functions with supports contained in $B_{R}$ for some $R>0$ such that $u_{1}$, $v_{1}$ are not identically zero. Let $(u, v)$ be the local (in time) energy solution to the weakly coupled system (1.1) according to Definition 2.1. Then, these solutions fulfill

$$
\operatorname{supp} u, \operatorname{supp} v \subset\left\{(t, x) \in[0, T) \times \mathbb{R}^{n}:|x| \leqslant R+t\right\}
$$

and the solution $(u, v)$ blows up in finite time, i.e. $T<\infty$.

Remark 2.1. Indeed, the assumption $g_{k}^{\prime}(t) \leqslant 0$ can be replaced by: $g_{k}(t) \gtrsim \tilde{g}_{k}(t)>0$ for all $t \geqslant 0$, where $\tilde{g}_{k}^{\prime}(t) \leqslant 0$. One may see the explanation in Remark 4.1 later. Thus, it can solve a more general case even it has oscillations. For instance, we can choose $g_{k}(t)=(3+2 \sin t) t^{-\gamma}$ with $\gamma \in[0,1)$ so that $g_{k}(t) \geqslant \tilde{g}_{k}(t)=t^{-\gamma}$. Here, we restricted $\gamma<1$ since the local existence result holds if $g_{k}(t) \in L^{1}([0, T])$.

Remark 2.2. We may observe from Theorem 2.2 that the decay function $t^{-1}$ somehow is the threshold of memory kernels on the blow-up result. Precisely, providing that the memory kernels 
decay slower than $t^{-1}$, we may notice the interplay effect between $g_{1}(t)$ as well as $g_{2}(t)$ in the blowup condition (2.3). Nevertheless, if the memory kernels decay faster than $t^{-1}$, then they do not influence on the blow-up condition (2.4) any more, which coincides with those for weakly coupled systems (1.4). We should underline that the condition (2.3) continuous to (2.4) as $g_{k}(t) \rightarrow t^{-1}$ for $k=1,2$. What's more, we have obtained the result not only for $g_{k}(t) \gtrsim t^{-1}$ but also for $g_{k}(t) \lesssim t^{-1}$, which means that the blow-up result stated in Theorem 2.2 covers a general class of memory kernels belonging to $L^{1}([0, T])$.

Remark 2.3. Let $p, q>1$ such that $p, q \leqslant n /(n-2)$ when $n \geqslant 3$. Concerning the case that only one memory kernel decreases slower than $t^{-1}$, namely,

$$
\min \left\{g_{1}(t), g_{2}(t)\right\} \lesssim t^{-1} \lesssim \max \left\{g_{1}(t), g_{2}(t)\right\} \text { for any } t \geqslant t_{0}
$$

where $t_{0} \in[0, T)$, we believe the energy solutions $(u, v)$ of the weakly coupled systems (1.1) blows up in finite time, i.e. $T<\infty$, if $g_{k}(t) \in L^{1}([0, T])$ for $k=1,2$ such that

- $g_{1}(t) \in \mathscr{C}^{2}([0, T])$ with $g_{1}^{\prime \prime}(0)>0, g_{2}^{\prime}(t) \leqslant 0$ and we consider $(p, q)$ so that the next estimate is satisfied:

$$
g_{2}(t) \max \left\{t^{1-q+2 q+1 / p},\left(g_{2}(t)\right)^{p-1} t^{2 p+1 / q}\right\} \gtrsim t^{\frac{n-1}{2}(p q-1)-2} \mathscr{L}(t)
$$

when $g_{1}(t) \lesssim t^{-1} \lesssim g_{2}(t)$ for any $t \geqslant t_{0}$

- $g_{2}(t) \in \mathscr{C}^{2}([0, T])$ with $g_{2}^{\prime \prime}(0)>0, g_{1}^{\prime}(t) \leqslant 0$ and we consider $(p, q)$ so that the next estimate $i s$ satisfied:

$$
g_{1}(t) \max \left\{\left(g_{1}(t)\right)^{q-1} t^{2 q+1 / p}, t^{1-p+2 p+1 / q}\right\} \gtrsim t^{\frac{n-1}{2}(p q-1)-2} \mathscr{L}(t),
$$

when $g_{2}(t) \lesssim t^{-1} \lesssim g_{1}(t)$ for any $t \geqslant t_{0}$

under the assumption that $\left(u_{0}, u_{1}, v_{0}, v_{1}\right) \in\left(H^{1}\left(\mathbb{R}^{n}\right) \times L^{2}\left(\mathbb{R}^{n}\right)\right) \times\left(H^{1}\left(\mathbb{R}^{n}\right) \times L^{2}\left(\mathbb{R}^{n}\right)\right)$ are nonnegative and compactly supported functions with supports contained in $B_{R}$ for some $R>0$ such that $u_{1}, v_{1}$ are not identically zero. We expect this conjecture can be proved by combining the proof of Case 1 and Case 2 in Section 4 without any additional technical difficulties. Precisely, to treat the nonlinear term containing $\min \left\{g_{1}(t), g_{2}(t)\right\}$, one may directly repeat the step of those in Case 1 . On the other hand, to deal with the nonlinear term containing $\max \left\{g_{1}(t), g_{2}(t)\right\}$, one may follow the analogous procedure to those stated in Case 2.

Remark 2.4. The construction of the function $\mathscr{L}(t)$ on the right-hand side of the condition (2.3) is used to guarantee

$$
g_{1}(t) g_{2}(t) \max \left\{\left(g_{1}(t)\right)^{q-1} t^{2 q+1 / p},\left(g_{2}(t)\right)^{p-1} t^{2 p+1 / q}\right\} t^{-\frac{n-1}{2}(p q-1)+3} \geqslant C_{\text {Suit }}
$$

for any $t \geqslant t_{\text {Suit }}$, where $t_{\text {Suit }} \geqslant 0$ is a suitable constant and $C_{\text {Suit }}=C_{\text {Suit }}\left(u_{0}, u_{1}, v_{0}, v_{1}, n, p, R, p, q\right)$ is a suitable positive constant. Indeed, due to the fact that by fixing the size of initial data, dimension, radius of the support of initial data and the power exponents of the nonlinearities, i.e. we determined the constant $C_{\text {Suit }}$, there exists a nonnegative constant $t_{\text {Suit }}$ such that

$$
\mathscr{L}(t) \geqslant C_{\text {Suit }}(n, p, R, p, q)
$$

for any $t \geqslant t_{\text {Suit }}$, where we used the property that $\lim _{t \rightarrow \infty} \mathscr{L}(t)=\infty$. 
Remark 2.5. Indeed, the condition $g_{k}(t) \in L^{1}([0, T])$ for the case when $g_{k}(t) \lesssim t^{-1}$ for $t \geqslant t_{0}$ with $t_{0} \in[0, T)$ is trivial. Because of the continuity of $g_{k}(t)$ for $t \in\left[0, \max \left\{t_{0}, \tilde{t}_{0}\right\}\right]$ with $\tilde{t}_{0} \in(0, T)$, we obtain $g_{k}(t) \lesssim 1$ for any $t \in\left[0, \max \left\{t_{0}, \tilde{t}_{0}\right\}\right]$. Thus,

$$
\int_{0}^{T} g_{k}(t) \mathrm{d} t=\int_{0}^{\max \left\{t_{0}, \tilde{t}_{0}\right\}} g_{k}(t) \mathrm{d} t+\int_{\max \left\{t_{0}, \tilde{t}_{0}\right\}}^{T} g_{k}(t) \mathrm{d} t \lesssim \max \left\{t_{0}, \tilde{t}_{0}\right\}+\ln (T)-\ln \left(\max \left\{t_{0}, \tilde{t}_{0}\right\}\right) .
$$

In other words, one derives $g_{k}(t) \in L^{1}([0, T])$ for any $k=1,2$.

As we known in the previous studies on the weakly coupled systems and single semilinear equations, it is clear that in the special case when the weakly coupled systems carrying $p=q$, the result is wholly symmetric to what occurs in the case of a single semilinear equation. Then, we can get the next result by taking $p=q$ and $g(t)=g_{1}(t)=g_{2}(t)$ in the last theorem, which also can be proved by strictly following the procedure of those for Theorem 2.2. What's more, similarly to Theorem 2.1, under the assumption $g(t) \in L^{1}([0, T])$, the local (in time) solution to the single semilinear equation uniquely exists if $p>1$ when $n=1,2$ and $1<p \leqslant n /(n-2)$ when $n \geqslant 3$.

Corollary 2.1. Let $p>1$ such that $p \leqslant n /(n-2)$ when $n \geqslant 3$. Let us consider $g(t) \in L^{1}([0, T])$ such that one of the following condition holds:

- if $g(t) \gtrsim t^{-1}$ for any $t \geqslant t_{0}$ with $t_{0} \in[0, T)$ and $g^{\prime}(t) \leqslant 0$, then we consider $(p, q)$ so that the next estimate is satisfied:

$$
g(t) \gtrsim t^{\frac{n-1}{2}(p-1)-2-\frac{1}{p}} \mathscr{L}(t)
$$

- if $g(t) \lesssim t^{-1}$ for any $t \geqslant t_{0}$ with $t_{0} \in[0, T)$ and $g(t) \in \mathscr{C}^{2}([0, T])$ with $g^{\prime \prime}(0)>0$, then we consider $(p, q)$ so that the next estimate is satisfied:

$$
1<p<p_{\mathrm{Str}}(n)
$$

where $p_{\text {Str }}(n)$ denotes the Strauss exponent and it has been defined in the introduction.

Let us suppose that $\left(u_{0}, u_{1}\right) \in H^{1}\left(\mathbb{R}^{n}\right) \times L^{2}\left(\mathbb{R}^{n}\right)$ are nonnegative and compactly supported functions with supports contained in $B_{R}$ for some $R>0$ such that $u_{1}$ is not identically zero. Let $u$ be the local (in time) energy solution to

$$
\begin{cases}u_{t t}-\Delta u=g *|u|^{p}, & x \in \mathbb{R}^{n}, t>0, \\ \left(u, u_{t}\right)(0, x)=\left(u_{0}, u_{1}\right)(x), & x \in \mathbb{R}^{n} .\end{cases}
$$

Then, these solutions fulfill

$$
\operatorname{supp} u \subset\left\{(t, x) \in[0, T) \times \mathbb{R}^{n}:|x| \leqslant R+t\right\}
$$

and the solution $u$ blows up in finite time, i.e. $T<\infty$.

Remark 2.6. Actually, the local (in time) energy solution of (2.8) has the similar definition to those in Definition 2.1 such that

$$
u \in \mathscr{C}\left([0, T), H^{1}\left(\mathbb{R}^{n}\right)\right) \cap \mathscr{C}^{1}\left([0, T), L^{2}\left(\mathbb{R}^{n}\right)\right) \text { with } g *|u|^{p} \in L_{\mathrm{loc}}^{1}\left([0, T) \times \mathbb{R}^{n}\right)
$$


fulfills $u(0, \cdot)=u_{0}$ in $H^{1}\left(\mathbb{R}^{n}\right)$ and the integral relation

$$
\begin{aligned}
& \int_{0}^{t} \int_{\mathbb{R}^{n}}\left(-u_{t}(s, x) \phi_{s}(s, x)+\nabla u(s, x) \cdot \nabla \phi(s, x)\right) \mathrm{d} x \mathrm{~d} s+\int_{\mathbb{R}^{n}} u_{t}(t, x) \phi(t, x) \mathrm{d} x \\
& =\int_{\mathbb{R}^{n}} u_{1}(x) \phi(0, x) \mathrm{d} x+\int_{0}^{t} \int_{\mathbb{R}^{n}}\left(g *|u|^{p}\right)(s, x) \phi(s, x) \mathrm{d} x \mathrm{~d} s
\end{aligned}
$$

for any test function $\phi \in \mathscr{C}_{0}^{\infty}\left([0, T) \times \mathbb{R}^{n}\right)$ and any $t \in(0, T)$.

Remark 2.7. We should point out that if $g_{1}(t), g_{2}(t)$ are replaced by $g(t)$, then Remarks 2.1, 2.2 and 2.4 also hold for Corollary 2.1.

\subsection{Application of Corollary 2.1}

In this subsection, we will give some applications (examples) of Corollary 2.1 by taking some special kinds of memory kernels. These applications can be coincided with the developed results in $[3,2]$. Throughout this part, we assume $p>1$ such that $p \leqslant n /(n-2)$ when $n \geqslant 3$.

Example 2.1. Let us consider the semilinear wave equation (2.8) with the Riemann-Liouville fractional integrals of order $1-\gamma$ and $\gamma \in(0,1)$, that is the nonlinear term (1.3). Obviously, it holds that $g(t) \gtrsim t^{-1}$ for any $t \geqslant 0$ and $g(t) \in L^{1}([0, T])$. We now employ Corollary 2.1 with the condition (2.6) leading to

$$
(n-1) p^{2}-(n+3-2 \gamma) p-2<0
$$

In other words, let us assume initial data satisfying the same assumptions as those in Corollary 2.1 and $1<p<p_{0}(n, \gamma)$, where $p_{0}(n, \gamma)$ stands for a generalized Strauss exponent defined in the introduction. Then, every energy solution of the semilinear wave equation (1.2) carrying (1.3) blows up in finite time. Thus, this result coincides with Theorem 1 in [2].

Example 2.2. Let us consider the semilinear wave equation (2.8) with exponential decay memory kernel $g(t)=\mathrm{e}^{-t / \beta}$ with $\beta>0$. Note that $g(t) \lesssim t^{-1}$ for $t \geqslant t_{0}(\beta)$. From Corollary 2.1, the blow-up condition (2.7), exactly, is $1<p<p_{\text {Str }}(n)$. Thanks to the derivative relation for the exponential decay function that

$$
\partial_{t}\left(g *|u|^{p}\right)(t, x)=|u(t, x)|^{p}-\frac{1}{\beta}\left(g *|u|^{p}\right)(t, x),
$$

we may transfer the Cauchy problem in this example into a special kind of semilinear Moore-GibsonThompson equations in the conservative case describing acoustic waves (one may the detail introduction in the recent paper [28]), namely,

$$
\begin{cases}\beta u_{t t t}+u_{t t}-\Delta u-\beta \Delta u_{t}=\beta|u|^{p}, & x \in \mathbb{R}^{n}, t>0 \\ \left(u, u_{t}, u_{t t}\right)(0, x)=\left(u_{0}, u_{1}, \Delta u_{0}\right)(x), & x \in \mathbb{R}^{n}\end{cases}
$$

Indeed, the deduction of the previous statement was motived by

$$
\begin{aligned}
\beta u_{t t t}+u_{t t}-\Delta u-\beta \Delta u_{t} & =\left(\beta \partial_{t}+\mathscr{I}\right)\left(u_{t t}-\Delta u\right), \\
\beta|u|^{p} & =\left(\beta \partial_{t}+\mathscr{I}\right)\left(g *|u|^{p}\right),
\end{aligned}
$$


where $\mathscr{I}$ is the identity operator such that $\mathscr{I}: f \rightarrow \mathscr{I} f \equiv f$. The recent paper [3] proved blow-up for the semilinear Moore-Gibson-Thompson equations in the conservative case if $1<p<p_{\text {Str }}(n)$, which somehow coincides with our result in this example.

Example 2.3. Let us consider the semilinear wave equation (2.8) with a very fast decay memory kernel such that

$$
g(t)=\exp (\exp (\cdots \exp (-c t))) \text { with } c>0 .
$$

Due to the fact that $g(t) \lesssim t^{-1}$ for $t \geqslant t_{0}(c)$. We still can derive blow-up of solutions from Corollary 2.1 if the power of the exponent fulfills $1<p<p_{\mathrm{Str}}(n)$.

\subsection{Application of Theorem 2.2}

In this subsection, we will show several applications on Theorem 2.2 by considering slow decay memory kernels and fast decay memory kernels, respectively. Throughout this part, we assume $p, q>1$ such that $p, q \leqslant n /(n-2)$ when $n \geqslant 3$.

Example 2.4. Let us consider the weakly coupled systems (1.1) with nonlinear memory terms being the Riemann-Liouville fractional integrals of order $1-\gamma_{1}$ and $1-\gamma_{2}$, respectively, where $\gamma_{1}, \gamma_{2} \in(0,1)$. Precisely, we take

$$
g_{1}(t)=\frac{t^{-\gamma_{1}}}{\Gamma\left(1-\gamma_{1}\right)} \text { and } g_{2}(t)=\frac{t^{-\gamma_{2}}}{\Gamma\left(1-\gamma_{2}\right)} .
$$

Clearly, $g_{k}(t) \gtrsim t^{-1}$ and $g_{k}(t) \in L^{1}([0, T])$ for $k=1,2$ and any $t \geqslant 0$. We now employ Theorem 2.2 with the condition (2.3) such that one of the following inequalities hold:

$$
\begin{aligned}
& \frac{n-1}{2} p q-2 q-\frac{1}{p}-\frac{n+5}{2}+\gamma_{1} q+\gamma_{2}<0 \quad \text { iff } \frac{\left(2-\gamma_{1}\right) q+\left(3-\gamma_{2}\right)+p^{-1}}{p q-1}>\frac{n-1}{2}, \\
& \frac{n-1}{2} p q-2 p-\frac{1}{q}-\frac{n+5}{2}+\gamma_{1}+\gamma_{2} p<0 \quad \text { iff } \frac{\left(2-\gamma_{2}\right) p+\left(3-\gamma_{1}\right)+q^{-1}}{p q-1}>\frac{n-1}{2} .
\end{aligned}
$$

So, let us assume initial data satisfying the same assumptions as those in Theorem 2.2 and $\alpha_{\mathrm{WM}}\left(p, q, \gamma_{1}, \gamma_{2}\right)>$ $(n-1) / 2$, where

$$
\alpha_{\mathrm{WM}}\left(p, q, \gamma_{1}, \gamma_{2}\right):=\max \left\{\frac{\left(2-\gamma_{2}\right) p+\left(3-\gamma_{1}\right)+q^{-1}}{p q-1}, \frac{\left(2-\gamma_{1}\right) q+\left(3-\gamma_{2}\right)+p^{-1}}{p q-1}\right\} .
$$

Then, every energy solution according to Definition 2.1 of the weakly coupled system (1.1) carrying (2.9) blows up in finite time. Particularly, because the next relation holds in the sense of distribution:

$$
\lim _{\gamma \rightarrow 1^{-}} \frac{s_{+}^{-\gamma_{k}}}{\Gamma\left(1-\gamma_{k}\right)}=\delta_{0}(s) \quad \text { carrying } s_{+}^{-\gamma_{k}}:= \begin{cases}s^{-\gamma_{k}} & \text { if } s>0 \\ 0 & \text { if } s<0\end{cases}
$$

it seems suitable to prove the blow-up result with (2.10) satisfying

$$
\lim _{\gamma_{1} \rightarrow 1^{-}, \gamma_{2} \rightarrow 1^{-}} \alpha_{\mathrm{WM}}\left(p, q, \gamma_{1}, \gamma_{2}\right)=\alpha_{\mathrm{W}}(p, q),
$$

in which $\alpha_{\mathrm{W}}(p, q)$ describes the critical curve of the weakly coupled systems for wave equations (1.4). 
Example 2.5. Let us consider the weakly coupled systems (1.1) with nonlinear memory terms, which are polynomial decay (faster than those in Example 2.4) without any singularities at $t=0$. To be specific, we consider

$$
g_{1}(t)=(1+t)^{-\gamma_{1}} \quad \text { and } \quad g_{2}(t)=(1+t)^{-\gamma_{2}}
$$

where $\gamma_{1}, \gamma_{2} \in[1, \infty)$. By concerning the condition (2.4) and the same assumptions on initial data as those in Theorem 2.2, every energy solution $(u, v)$ blows up in finite time. Furthermore, if we consider the exponential decay memory kernels such that

$$
g_{1}(t)=\mathrm{e}^{-c_{1} t} \text { and } g_{2}(t)=\mathrm{e}^{-c_{2} t}
$$

with $c_{1}, c_{2}>0$, then the blow-up condition is still shown by (2.4). In the special case by following the same idea as Example 2.2, we take $c_{1}=1 / \beta_{1}>0$ and $c_{2}=1 / \beta_{2}>0$, every energy solution to the weakly coupled systems for the Moore-Gibson-Thompson equations

$$
\begin{cases}\beta_{1} u_{t t t}+u_{t t}-\Delta u-\beta_{1} \Delta u_{t}=\beta_{1}|v|^{p}, & x \in \mathbb{R}^{n}, t>0 \\ \beta_{2} v_{t t t}+v_{t t}-\Delta v-\beta_{2} \Delta v_{t}=\beta_{2}|u|^{q}, & x \in \mathbb{R}^{n}, t>0 \\ \left(u, u_{t}, u_{t t}, v, v_{t}, v_{t t}\right)(0, x)=\left(u_{0}, u_{1}, \Delta u_{0}, v_{0}, v_{1}, \Delta v_{0}\right)(x), & x \in \mathbb{R}^{n}\end{cases}
$$

blows up provided that the condition (2.4) holds.

\section{Proof of Theorem 2.1}

First of all, let us introduce some notations and well-developed results for linear wave equation. It is well-known that the solutions $w=w(t, x)$ of linear wave equation

$$
\begin{cases}w_{t t}-\Delta w=0, & x \in \mathbb{R}^{n}, t>0 \\ \left(w, w_{t}\right)(0, x)=\left(w_{0}, w_{1}\right)(x), & x \in \mathbb{R}^{n}\end{cases}
$$

is given by the form

$$
w(t, x)=\mathscr{K}_{0}(t, x) *(x) w_{0}(x)+\mathscr{K}_{1}(t, x) *(x) w_{1}(x),
$$

where we denoted by $\mathscr{K}_{0}(t, x)$ and $\mathscr{K}_{1}(t, x)$ the fundamental solutions to the linear wave equation in $\mathbb{R}^{n}$ with initial data $\left(w_{0}, w_{1}\right)=\left(\delta_{0}, 0\right)$ and $\left(w_{0}, w_{1}\right)=\left(0, \delta_{0}\right)$, respectively. Here, $\delta_{0}$ is the Dirac distribution in $x=0$ with respect to spatial variables. Precisely, the above kernels $\mathscr{K}_{0}(t, x)$ and $\mathscr{K}_{1}(t, x)$ may be represented by the application of partial Fourier transforms with respect to time variable as follows:

$$
\mathscr{K}_{0}(t, x)=\mathscr{F}_{\xi \rightarrow x}^{-1}(\cos (|\xi| t)) \text { and } \mathscr{K}_{1}(t, x)=\mathscr{F}_{\xi \rightarrow x}^{-1}\left(\frac{\sin (|\xi| t)}{|\xi|}\right) .
$$

Moreover, the solution of the Cauchy problem (3.11) fulfills the following $L^{2}-L^{2}$ estimates:

$$
\begin{aligned}
\|w(t, \cdot)\|_{L^{2}\left(\mathbb{R}^{n}\right)} & \lesssim\left\|w_{0}\right\|_{L^{2}\left(\mathbb{R}^{n}\right)}+(1+t)\left\|w_{1}\right\|_{L^{2}\left(\mathbb{R}^{n}\right)} \\
\|\nabla w(t, \cdot)\|_{L^{2}\left(\mathbb{R}^{n}\right)}+\left\|w_{t}(t, \cdot)\right\|_{L^{2}\left(\mathbb{R}^{n}\right)} & =\left\|w_{0}\right\|_{H^{1}\left(\mathbb{R}^{n}\right)}+\left\|w_{1}\right\|_{L^{2}\left(\mathbb{R}^{n}\right)} .
\end{aligned}
$$


These estimates can be proved easily by using the phase space analysis. One may check Chapter 10 of [11] in detail.

We define the solution space to (1.1) by

$$
\begin{gathered}
X(T):=\left\{(u, v) \in\left(\mathscr{C}\left([0, T], H^{1}\left(\mathbb{R}^{n}\right)\right) \cap \mathscr{C}^{1}\left([0, T], L^{2}\left(\mathbb{R}^{n}\right)\right)\right)^{2}\right. \text { such that } \\
\left.\quad \operatorname{supp} u(t, \cdot), \operatorname{supp} v(t, \cdot) \subset B_{R+t} \text { for any } t \in[0, T]\right\}
\end{gathered}
$$

carrying its norm

$$
\|(u, v)\|_{X(T)}:=\max _{t \in[0, T]}(\mathcal{M}[u](t)+\mathcal{M}[v](t)),
$$

where we defined for $w=u, v$ that

$$
\mathcal{M}[w](t):=\|w(t, \cdot)\|_{L^{2}\left(\mathbb{R}^{n}\right)}+\|\nabla w(t, \cdot)\|_{L^{2}\left(\mathbb{R}^{n}\right)}+\left\|w_{t}(t, \cdot)\right\|_{L^{2}\left(\mathbb{R}^{n}\right)} .
$$

By using Duhamel's principle, we may introduce the operator $N$ such that

$$
N:(u, v) \in X(T) \rightarrow N(u, v):=\left(u^{\operatorname{lin}}(t, x)+u^{\mathrm{non}}(t, x), v^{\operatorname{lin}}(t, x)+v^{\text {non }}(t, x)\right) .
$$

Here, we denote

$$
\begin{aligned}
u^{\operatorname{lin}}(t, x) & :=\mathscr{K}_{0}(t, x) *_{(x)} u_{0}(x)+\mathscr{K}_{1}(t, x) *_{(x)} u_{1}(x), \\
u^{\text {non }}(t, x) & :=\int_{0}^{t} \mathscr{K}_{1}(t-\tau, x) *_{(x)}\left(g_{1} *|v|^{p}\right)(\tau, x) \mathrm{d} \tau, \\
v^{\operatorname{lin}}(t, x) & :=\mathscr{K}_{0}(t, x) *{ }_{(x)} v_{0}(x)+\mathscr{K}_{1}(t, x) *(x) \\
v^{\text {non }}(t, x) & :=\int_{0}^{t} \mathscr{K}_{1}(t-\tau, x) *{ }_{(x)}\left(g_{2} *|u|^{q}\right)(\tau, x) \mathrm{d} \tau .
\end{aligned}
$$

Next, we are going to consider as mild local (in time) solution of (1.1) the fixed points of the operator $N$. In other words, we should prove

$$
\begin{gathered}
\|N(u, v)\|_{X(T)} \leqslant \bar{c}_{0}+\bar{c}_{1} T\left(\|(u, v)\|_{X(T)}^{p}+\|(u, v)\|_{X(T)}^{q}\right), \\
\|N(u, v)-N(\bar{u}, \bar{v})\|_{X(T)} \leqslant \bar{c}_{2} T\|(u, v)-(\bar{u}, \bar{v})\|_{X(T)} \sum_{r=p, q}\left(\|(u, v)\|_{X(T)}^{r-1}+\|(\bar{u}, \bar{v})\|_{X(T)}^{r-1}\right),
\end{gathered}
$$

where $\bar{c}_{k}=\bar{c}_{k}\left(u_{0}, u_{1}, v_{0}, v_{1}\right)>0$ for $k=1,2,3$, to showing the local (in time) existence and uniqueness of the solution in $X(T)$.

Obviously from the beginning of this section, we claim $\left(u^{\operatorname{lin}}, v^{\operatorname{lin}}\right) \in X(T)$ satisfying

$$
\left\|\left(u^{\operatorname{lin}}, v^{\operatorname{lin}}\right)\right\|_{X(T)} \lesssim\left\|\left(u_{0}, v_{0}\right)\right\|_{H^{1}\left(\mathbb{R}^{n}\right) \times H^{1}\left(\mathbb{R}^{n}\right)}+(1+T)\left\|\left(u_{1}, v_{1}\right)\right\|_{L^{2}\left(\mathbb{R}^{n}\right) \times L^{2}\left(\mathbb{R}^{n}\right)} .
$$

In order to arrive at (3.12), by employing the classical Gagliardo-Nirenberg inequality, one immediately derives

$$
\|w(\eta, \cdot)\|_{L^{2 r\left(\mathbb{R}^{n}\right)}}^{r} \leqslant c\|w(\eta, \cdot)\|_{L^{2}\left(\mathbb{R}^{n}\right)}^{\left(1-\frac{n}{r}\left(1-\frac{1}{r}\right)\right) r}\|\nabla w(\eta, \cdot)\|_{L^{2}\left(\mathbb{R}^{n}\right)}^{\frac{n}{2}\left(1-\frac{1}{r}\right) r} \leqslant c\|(u, v)\|_{X(T)}^{r},
$$

for $\eta \in[0, T]$, with $r>1$ if $n=1,2$ and $1<r \leqslant n /(n-2)$ if $n \geqslant 3$, where we denoted $w=u, v$ and $r=p, q$ in the last inequality. 
Then, combining obtained $L^{2}-L^{2}$ estimates and the previous $L^{2 r}$ estimates of solutions, we have

$$
\begin{aligned}
\left\|\int_{0}^{t} \mathscr{K}_{1}(t-\tau, x) *_{(x)}\left(g_{1} *|v|^{p}\right)(\tau, x)\right\|_{L^{2}\left(\mathbb{R}^{n}\right)} & \leqslant c \int_{0}^{t}(1+t-\tau) \int_{0}^{\tau} g_{1}(\tau-\eta)\left\||v(\eta, \cdot)|^{p}\right\|_{L^{2}\left(\mathbb{R}^{n}\right)} \mathrm{d} \eta \mathrm{d} \tau \\
& \leqslant c \int_{0}^{t}(1+t-\tau) \int_{0}^{\tau} g_{1}(\tau-\eta) \mathrm{d} \eta \mathrm{d} \tau\|(u, v)\|_{X(T)}^{p} \\
& \leqslant c \int_{0}^{t}(1+t-\tau) \mathrm{d} \tau\|(u, v)\|_{X(T)}^{p} \\
& \leqslant c(1+t) t\|(u, v)\|_{X(T)}^{p},
\end{aligned}
$$

where we used $g_{1}(t) \in L^{1}([0, T])$, namely, for $[0, \tau] \subset[0, T]$,

$$
\int_{0}^{\tau} g_{1}(\tau-\eta) \mathrm{d} \eta=\int_{0}^{\tau} g_{1}(\eta) \mathrm{d} \eta \leqslant \int_{0}^{T} g_{1}(t) \mathrm{d} t<\infty .
$$

Analogously,

$$
\left\|\int_{0}^{t} \mathscr{K}_{1}(t-\tau, x) *(x)\left(g_{2} *|u|^{q}\right)(\tau, x)\right\|_{L^{2}\left(\mathbb{R}^{n}\right)} \leqslant c(1+t) t\|(u, v)\|_{X(T)}^{q},
$$

where we applied our assumption $g_{2}(t) \in L^{1}([0, T])$.

By the same way, we derived

$$
\begin{aligned}
& \left\|\nabla^{j} \partial_{t}^{k} \int_{0}^{t} \mathscr{K}_{1}(t-\tau, x) *_{(x)}\left(g_{1} *|v|^{p}\right)(\tau, x) \mathrm{d} \tau\right\|_{L^{2}\left(\mathbb{R}^{n}\right)} \leqslant c t\|(u, v)\|_{X(T)}^{p}, \\
& \left\|\nabla^{j} \partial_{t}^{k} \int_{0}^{t} \mathscr{K}_{1}(t-\tau, x) *_{(x)}\left(g_{2} *|u|^{q}\right)(\tau, x) \mathrm{d} \tau\right\|_{L^{2}\left(\mathbb{R}^{n}\right)} \leqslant c t\|(u, v)\|_{X(T)}^{q},
\end{aligned}
$$

for any $j, k \in \mathbb{N}_{0}$ such that $j+k=1$. They implies our desired inequality (3.12).

Furthermore, the function $N(u, v)$ is the solution of the Cauchy problem for wave equations

$$
\begin{cases}\partial_{t}^{2} N(u, v)-\Delta N(u, v)=\left(\left(g_{1} *|v|^{p}\right),\left(g_{2} *|u|^{q}\right)\right), & x \in \mathbb{R}^{n}, t>0, \\ \left(N(u, v), \partial_{t} N(u, v)\right)(0, x)=\left(u_{0}, u_{1}, v_{0}, v_{1}\right)(x), & x \in \mathbb{R}^{n} .\end{cases}
$$

We claim that $\operatorname{supp} N(u, v)(t, \cdot) \subset B_{R+t} \times B_{R+t}$ for any $t \in[0, T]$ because $(u, v)$ is assumed to be supported in the forward cone. Therefore, it is proved that the operator $N$ maps $X(T)$ into itself.

Finally, by considering $w=u, v$ and $r=p, q$, with the aid of

$$
\left.|| w(\tau, x)\right|^{r}-|\bar{w}(\tau, x)|^{r}|\leqslant c| w(\tau, x)-\bar{w}(\tau, x) \mid\left(|w(\tau, x)|^{r-1}+|\bar{w}(\tau, x)|^{r-1}\right),
$$

we are able to gain from Hölder's inequality that

$$
\left\||w(\tau, \cdot)|^{r}-|\bar{w}(\tau, \cdot)|^{r}\right\|_{L^{2}\left(\mathbb{R}^{n}\right)} \leqslant c\|w(\tau, \cdot)-\bar{w}(\tau, \cdot)\|_{L^{2}\left(\mathbb{R}^{n}\right)}\left(\|w(\tau, \cdot)\|_{L^{2 r}\left(\mathbb{R}^{n}\right)}^{r-1}+\|\bar{w}(\tau, \cdot)\|_{L^{2 r}\left(\mathbb{R}^{n}\right)}^{r-1}\right) .
$$

We know the equality holds

$$
\|N(u, v)-N(\bar{u}, \bar{v})\|_{X(t)}=\left\|\int_{0}^{t} \mathscr{K}_{1}(t-\tau, x) *_{(x)}\left(F_{p}(\tau, x), F_{q}(\tau, x)\right) \mathrm{d} \tau\right\|_{X(t)},
$$


where we denoted

$$
\begin{aligned}
& F_{p}(\tau, x):=\int_{0}^{\tau} g_{1}(\tau-\eta)\left(|v(\eta, x)|^{p}-|\bar{v}(\eta, x)|^{p}\right) \mathrm{d} \eta \\
& F_{q}(\tau, x):=\int_{0}^{\tau} g_{2}(\tau-\eta)\left(|u(\eta, x)|^{q}-|\bar{u}(\eta, x)|^{q}\right) \mathrm{d} \eta .
\end{aligned}
$$

Similarly to the deduction of (3.12), we use $L^{2}-L^{2}$ estimates, which allows us to conclude (3.13). The proof is complete.

\section{Proof of Theorem 2.2}

To begin with the proof, let us first define time-dependent functionals related to the solutions

$$
U(t):=\int_{\mathbb{R}^{n}} u(t, x) \mathrm{d} x \text { and } V(t):=\int_{\mathbb{R}^{n}} v(t, x) \mathrm{d} x .
$$

In order to prove blow-up of energy solutions of the weakly coupled systems (1.1), we will show that the functional $(U(t), V(t))$ blows up in finite time by applying iteration methods. For this reason, we will construct a pair of coupled of integral inequalities for the spatial averages of the components of a local (in time) solution $(u, v)$ by choosing special test functions in the definition of energy solutions, which is so-called iteration frame, and derive first lower bound estimates for two functionals. Then, sharper estimates for the functionals from the below will be established by employing suitable iteration argument.

Here, we take the test functions in $(2.1)$ and $(2.2)$ such that $\phi \equiv 1 \equiv \psi$ in $\left\{(s, x) \in[0, t] \times \mathbb{R}^{n}\right.$ : $|x| \leqslant R+s\}$, which leads to

$$
\begin{gathered}
\int_{\mathbb{R}^{n}} u_{t}(t, x) \mathrm{d} x-\int_{\mathbb{R}^{n}} u_{1}(x) \mathrm{d} x=\int_{0}^{t} \int_{\mathbb{R}^{n}} \int_{0}^{s} g_{1}(s-\tau)|v(\tau, x)|^{p} \mathrm{~d} \tau \mathrm{d} x \mathrm{~d} s, \\
\int_{\mathbb{R}^{n}} v_{t}(t, x) \mathrm{d} x-\int_{\mathbb{R}^{n}} v_{1}(x) \mathrm{d} x=\int_{0}^{t} \int_{\mathbb{R}^{n}} \int_{0}^{s} g_{2}(s-\tau)|u(\tau, x)|^{q} \mathrm{~d} \tau \mathrm{d} x \mathrm{~d} s .
\end{gathered}
$$

The definition (4.1) allows us to rewrite the above relations into

$$
\begin{aligned}
& U^{\prime}(t)=U^{\prime}(0)+\int_{0}^{t} \int_{\mathbb{R}^{n}} \int_{0}^{s} g_{1}(s-\tau)|v(\tau, x)|^{p} \mathrm{~d} \tau \mathrm{d} x \mathrm{~d} s, \\
& V^{\prime}(t)=V^{\prime}(0)+\int_{0}^{t} \int_{\mathbb{R}^{n}} \int_{0}^{s} g_{2}(s-\tau)|u(\tau, x)|^{q} \mathrm{~d} \tau \mathrm{d} x \mathrm{~d} s .
\end{aligned}
$$

From the nonnegative assumptions on $u_{0}, u_{1}, v_{0}, v_{1}$ showing the nonnegativities of $U(0), U^{\prime}(0), V(0)$, $V^{\prime}(0)$, we are able to integrate $(4.2)$ and $(4.3)$, respectively, over $[0, t]$ to obtain

$$
\begin{aligned}
& U(t) \geqslant \int_{0}^{t} \int_{0}^{\eta} \int_{0}^{s} g_{1}(s-\tau) \int_{\mathbb{R}^{n}}|v(\tau, x)|^{p} \mathrm{~d} x \mathrm{~d} \tau \mathrm{d} s \mathrm{~d} \eta \geqslant 0, \\
& V(t) \geqslant \int_{0}^{t} \int_{0}^{\eta} \int_{0}^{s} g_{2}(s-\tau) \int_{\mathbb{R}^{n}}|u(\tau, x)|^{q} \mathrm{~d} x \mathrm{~d} \tau \mathrm{d} s \mathrm{~d} \eta \geqslant 0 .
\end{aligned}
$$

With the help of Hölder's inequality combined with the support conditions of solutions, there exist positive constants $C_{0}=C_{0}(n, R, p)$ and $\widetilde{C}_{0}=\widetilde{C}_{0}(n, R, q)$ such that

$$
\begin{aligned}
& \int_{\mathbb{R}^{n}}|v(\tau, x)|^{p} \mathrm{~d} x=\int_{B_{R+\tau}}|v(\tau, x)|^{p} \mathrm{~d} x \geqslant C_{0}(R+\tau)^{-n(p-1)}(V(\tau))^{p}, \\
& \int_{\mathbb{R}^{n}}|u(\tau, x)|^{q} \mathrm{~d} x=\int_{B_{R+\tau}}|u(\tau, x)|^{q} \mathrm{~d} x \geqslant \widetilde{C}_{0}(R+\tau)^{-n(q-1)}(U(\tau))^{q} .
\end{aligned}
$$


Therefore, the coupled system of crucial integral inequalities

$$
\begin{aligned}
& U(t) \geqslant C_{0} \int_{0}^{t} \int_{0}^{\eta} \int_{0}^{s} g_{1}(s-\tau)(R+\tau)^{-n(p-1)}(V(\tau))^{p} \mathrm{~d} \tau \mathrm{d} s \mathrm{~d} \eta, \\
& V(t) \geqslant \widetilde{C}_{0} \int_{0}^{t} \int_{0}^{\eta} \int_{0}^{s} g_{2}(s-\tau)(R+\tau)^{-n(q-1)}(U(\tau))^{q} \mathrm{~d} \tau \mathrm{d} s \mathrm{~d} \eta,
\end{aligned}
$$

hold for any $t \geqslant 0$. In other words, the iteration frames were constructed in (4.4) and (4.5).

Our second step is to derive first lower bound estimates for the functionals $U(t)$ and $V(t)$, respectively. Strongly motivated by the pioneering research [34], we introduce the eigenfunction $\Phi=\Phi(x)$ of the Laplace operator in $n$ dimensional whole space, i.e.,

$$
\begin{array}{ll}
\Phi(x):=\mathrm{e}^{x}+\mathrm{e}^{-x} & \text { if } n=1, \\
\Phi(x):=\int_{\mathbb{S}^{n-1}} \mathrm{e}^{x \cdot \omega} \mathrm{d} \sigma_{\omega} & \text { if } n \geqslant 2,
\end{array}
$$

where $\mathbb{S}^{n-1}$ is the $n-1$ dimensional sphere, which is devoted to the definition of the test function $\Psi=\Psi(t, x)$ such that $\Psi(t, x)=\mathrm{e}^{-t} \Phi(x)$. Plainly, the function $\Psi$ is the solution of the homogeneous wave equation $\Psi_{t t}-\Delta \Psi=0$. To investigate the estimates for $U(t)$ and $V(t)$ from the below, let us now define two auxiliary functionals

$$
U_{0}(t):=\int_{\mathbb{R}^{n}} u(t, x) \Psi(t, x) \mathrm{d} x \text { and } V_{0}(t):=\int_{\mathbb{R}^{n}} v(t, x) \Psi(t, x) \mathrm{d} x .
$$

Differentiating (4.2) and (4.3) with respective to time variable and employing Hölder's inequality, compactness of the support of the solutions to have

$$
\begin{aligned}
U^{\prime \prime}(t) & =\int_{0}^{t} g_{1}(t-\tau) \int_{\mathbb{R}^{n}}|v(\tau, x)|^{p} \mathrm{~d} x \mathrm{~d} \tau \geqslant C_{1} \int_{0}^{t} g_{1}(t-\tau)(R+\tau)^{n-1-\frac{n-1}{2} p}\left|V_{0}(\tau)\right|^{p} \mathrm{~d} \tau, \\
V^{\prime \prime}(t) & =\int_{0}^{t} g_{2}(t-\tau) \int_{\mathbb{R}^{n}}|u(\tau, x)|^{q} \mathrm{~d} x \mathrm{~d} \tau \geqslant \widetilde{C}_{1} \int_{0}^{t} g_{2}(t-\tau)(R+\tau)^{n-1-\frac{n-1}{2} q}\left|U_{0}(\tau)\right|^{q} \mathrm{~d} \tau,
\end{aligned}
$$

with suitable positive constants $C_{1}=C_{1}(n, R, p)$ and $\widetilde{C}_{1}=\widetilde{C}_{1}(n, R, q)$. Here, we apply the asymptotic behavior of $\Psi$ to gain

$$
\int_{B_{R+\tau}}|\Psi(\tau, x)|^{\frac{r}{r-1}} \mathrm{~d} x \lesssim(R+\tau)^{(n-1)\left(1-\frac{r^{\prime}}{2}\right)},
$$

where $r^{\prime}$ is the conjugate of $r$, i.e. $1 / r+1 / r^{\prime}=1$. One also may find (4.8) in [24].

The nonnegativities of the nonlinearities of the equations in (1.1) imply immediately from [34] the lower bound of $U_{0}(t)$ and $V_{0}(t)$ that

$$
\begin{aligned}
& U_{0}(t) \geqslant \frac{1-\mathrm{e}^{-2 t}}{2} \int_{\mathbb{R}^{n}} u_{0}(x) \Phi(x) \mathrm{d} x+\frac{1+\mathrm{e}^{-2 t}}{2} \int_{\mathbb{R}^{n}} u_{1}(x) \Phi(x) \mathrm{d} x \geqslant C_{2}, \\
& V_{0}(t) \geqslant \frac{1-\mathrm{e}^{-2 t}}{2} \int_{\mathbb{R}^{n}} v_{0}(x) \Phi(x) \mathrm{d} x+\frac{1+\mathrm{e}^{-2 t}}{2} \int_{\mathbb{R}^{n}} v_{1}(x) \Phi(x) \mathrm{d} x \geqslant \widetilde{C}_{2},
\end{aligned}
$$

for any $t \geqslant 0$ with suitable constants $C_{2}>0$ and $\widetilde{C}_{2}>0$ depending on the size of initial data, where we used our assumptions on nontrivial data $u_{1}$ as well as $v_{1}$. In this step, one needs to employ the 
relations $\Psi_{t}(t, x)=-\Psi(t, x)$ and $\Delta \Psi(t, x)=\Psi(t, x)$.

Consequently, according to (4.6), (4.7), they lead to

$$
\begin{aligned}
& U(t) \geqslant C_{1} \widetilde{C}_{2}^{p} \int_{0}^{t} \int_{0}^{\eta} \int_{0}^{s} g_{1}(s-\tau)(R+\tau)^{n-1-\frac{n-1}{2} p} \mathrm{~d} \tau \mathrm{d} s \mathrm{~d} \eta, \\
& V(t) \geqslant \widetilde{C}_{1} C_{2}^{q} \int_{0}^{t} \int_{0}^{\eta} \int_{0}^{s} g_{2}(s-\tau)(R+\tau)^{n-1-\frac{n-1}{2} q} \mathrm{~d} \tau \mathrm{d} s \mathrm{~d} \eta .
\end{aligned}
$$

To understand the precise lower bound for the functionals, we need to discuss the estimates under different assumptions on the memory kernels. To be specific, we will complete the proof by considering the next two cases separately:

- Case 1: $g_{k}(t) \gtrsim t^{-1}$ for any $t \geqslant t_{0}$ with $t_{0} \in[0, T)$ and all $k=1,2$;

- Case 2: $g_{k}(t) \lesssim t^{-1}$ for any $t \geqslant t_{0}$ with $t_{0} \in[0, T)$ and all $k=1,2$.

This criterion $t^{-1}$ is motivated by the fact that if the time-dependent function $g_{k}(t)$ decreases very fast, then the following lower bound estimate:

$$
\int_{0}^{t} g_{k}(t-\tau) \mathrm{d} \tau \gtrsim g_{k}(t) t \approx g_{k}(t)
$$

hold for any $t \gg 1$ and some losses appear, for example, the exponential decay memory kernel $g_{k}(t)=\mathrm{e}^{-t}$. It means that we will employ different approaches to derive lower bound estimates of the integral including the memory kernels, which primarily determined by the decreasing rate of the memory kernel.

\subsection{Treatment for Case 1}

In this case, we just need to directly apply the non-increasing properties for both memory kernels for any $t \geqslant 0$ to catch from (4.9) and (4.10) that

$$
\begin{aligned}
& U(t) \geqslant \frac{C_{1} \widetilde{C}_{2}^{p}}{n(n+1)(n+2)} g_{1}(t)(R+t)^{-\frac{n-1}{2} p} t^{n+2}, \\
& V(t) \geqslant \frac{\widetilde{C}_{1} C_{2}^{q}}{n(n+1)(n+2)} g_{2}(t)(R+t)^{-\frac{n-1}{2} q} t^{n+2} .
\end{aligned}
$$

Remark 4.1. Actually, one may generalize the assumption on $g_{k}(t)$ such that $g_{k}(t) \gtrsim \tilde{g}_{k}(t)>0$, where $\tilde{g}_{k}(t)$ is a constant or monotonously decreasing function. For example, from (4.9) we may directly arrive at

$$
\begin{aligned}
U(t) & \gtrsim C_{1} \widetilde{C}_{2}^{p}(R+\tau)^{-\frac{n-1}{2} p} \int_{0}^{t} \int_{0}^{\eta} \int_{0}^{s} \tilde{g}_{1}(s-\tau)(R+\tau)^{n-1} \mathrm{~d} \tau \mathrm{d} s \mathrm{~d} \eta \\
& \gtrsim C_{1} \widetilde{C}_{2}^{p} \tilde{g}_{1}(t)(R+\tau)^{-\frac{n-1}{2} p} \int_{0}^{t} \int_{0}^{\eta} \int_{0}^{s} \tau^{n-1} \mathrm{~d} \tau \mathrm{d} s \mathrm{~d} \eta \\
& \gtrsim \frac{C_{1} \widetilde{C}_{2}^{p}}{n(n+1)(n+2)} \tilde{g}_{1}(t)(R+t)^{-\frac{n-1}{2} p} t^{n+2} .
\end{aligned}
$$

Then, we just need to replace $g_{k}(t)$ by $\tilde{g}_{k}(t)$ in the next all steps. 
We derived the first lower bounds for the functionals such that

$$
\begin{aligned}
& U(t) \geqslant D_{1}\left(g_{1}(t)\right)^{a_{1}}\left(g_{2}(t)\right)^{\alpha_{1}}(R+t)^{-b_{1}} t^{\beta_{1}} \text { for any } t \geqslant 0, \\
& V(t) \geqslant \widetilde{D}_{1}\left(g_{1}(t)\right)^{\tilde{a}_{1}}\left(g_{2}(t)\right)^{\tilde{\alpha}_{1}}(R+t)^{-\tilde{b}_{1}} t^{\tilde{\beta}_{1}} \text { for any } t \geqslant 0 .
\end{aligned}
$$

Here, we denote constants in the previous estimates as

$$
\begin{aligned}
& D_{1}:=\frac{C_{1} \widetilde{C}_{2}^{p}}{n(n+1)(n+2)}, \quad \widetilde{D}_{1}:=\frac{\widetilde{C}_{1} C_{2}^{q}}{n(n+1)(n+2)}, \\
& a_{1}:=1, \quad \alpha_{1}:=0, \quad b_{1}:=\frac{n-1}{2} p, \quad \beta_{1}:=n+2, \\
& \tilde{a}_{1}:=0, \quad \tilde{\alpha}_{1}:=1, \quad \tilde{b}_{1}:=\frac{n-1}{2} q, \quad \tilde{\beta}_{1}:=n+2,
\end{aligned}
$$

In the forthcoming part, by the way of iteration argument we will prove sequences of lower bounds of $U(t)$ and $V(t)$ by combining the iteration frame (4.4) and (4.5) as follows:

$$
\begin{aligned}
& U(t) \geqslant D_{j}\left(g_{1}(t)\right)^{a_{j}}\left(g_{2}(t)\right)^{\alpha_{j}}(R+t)^{-b_{j}} t^{\beta_{j}} \text { for any } t \geqslant 0, \\
& V(t) \geqslant \widetilde{D}_{j}\left(g_{1}(t)\right)^{\tilde{a}_{j}}\left(g_{2}(t)\right)^{\tilde{\alpha}_{j}}(R+t)^{-\tilde{b}_{j}} t^{\tilde{\beta}_{j}} \text { for any } t \geqslant 0,
\end{aligned}
$$

where $\left\{D_{j}\right\}_{j \geqslant 1},\left\{\widetilde{D}_{j}\right\}_{j \geqslant 1},\left\{a_{j}\right\}_{j \geqslant 1},\left\{\tilde{a}_{j}\right\}_{j \geqslant 1},\left\{\alpha_{j}\right\}_{j \geqslant 1},\left\{\tilde{\alpha}_{j}\right\}_{j \geqslant 1},\left\{b_{j}\right\}_{j \geqslant 1},\left\{\tilde{b}_{j}\right\}_{j \geqslant 1},\left\{\beta_{j}\right\}_{j \geqslant 1}$ and $\left\{\tilde{\beta}_{j}\right\}_{j \geqslant 1}$ are sequences of nonnegative real numbers that will be determined iteratively in the inductive step. Note that the initial value, i.e. $j=0$, of the above inequalities are given in (4.11) and (4.12). We should clarify that we cannot automatically ignore the components of $g_{2}(t)$ in the lower bound sequence for $U(t)$ and of $g_{1}(t)$ in the lower bound sequence for $V(t)$ although $\alpha_{1}=\tilde{a}_{1}=0$. The main factor comes from the interaction in weakly coupled systems.

For this reason, by assuming the validities of (4.13) and (4.14) for $j$, we just need to prove the induction step, i.e. the validities of (4.13) and (4.14) for $j+1$. Let us now combine (4.13), (4.14) with $(4.5),(4.4)$ to arrive at

$$
\begin{aligned}
U(t) & \geqslant C_{0} \widetilde{D}_{j}^{p} \int_{0}^{t} \int_{0}^{\eta} \int_{0}^{s} g_{1}(s-\tau)\left(g_{1}(\tau)\right)^{\tilde{a}_{j} p}\left(g_{2}(\tau)\right)^{\tilde{\alpha}_{j} p}(R+\tau)^{-n(p-1)-\tilde{b}_{j} p} \tau^{\tilde{\beta}_{j} p} \mathrm{~d} \tau \mathrm{d} s \mathrm{~d} \eta \\
& \geqslant \frac{C_{0} \widetilde{D}_{j}^{p}}{\left(1+\tilde{\beta}_{j} p\right)\left(2+\tilde{\beta}_{j} p\right)\left(3+\tilde{\beta}_{j} p\right)}\left(g_{1}(t)\right)^{1+\tilde{a}_{j} p}\left(g_{2}(t)\right)^{\tilde{\alpha}_{j} p}(R+t)^{-n(p-1)-\tilde{b}_{j} p} t^{3+\tilde{\beta}_{j} p},
\end{aligned}
$$

and similarly,

$$
\begin{aligned}
V(t) & \geqslant \widetilde{C}_{0} D_{j}^{q} \int_{0}^{t} \int_{0}^{\eta} \int_{0}^{s}\left(g_{1}(\tau)\right)^{a_{j} q} g_{2}(s-\tau)\left(g_{2}(\tau)\right)^{\alpha_{j} q}(R+\tau)^{-n(q-1)-b_{j} q} \tau^{\beta_{j} q} \mathrm{~d} \tau \mathrm{d} s \mathrm{~d} \eta \\
& \geqslant \frac{\widetilde{C}_{0} D_{j}^{q}}{\left(1+\beta_{j} q\right)\left(2+\beta_{j} q\right)\left(3+\beta_{j} q\right)}\left(g_{1}(t)\right)^{a_{j} q}\left(g_{2}(t)\right)^{1+\alpha_{j} q}(R+t)^{-n(q-1)-b_{j} q} t^{3+\beta_{j} q} .
\end{aligned}
$$

Namely, the desired estimates (4.13) and (4.14) hold for $j+1$ providing that

$$
\begin{aligned}
& D_{j+1}:=\frac{C_{0} \widetilde{D}_{j}^{p}}{\left(1+\tilde{\beta}_{j} p\right)\left(2+\tilde{\beta}_{j} p\right)\left(3+\tilde{\beta}_{j} p\right)}, \quad \widetilde{D}_{j+1}:=\frac{\widetilde{C}_{0} D_{j}^{q}}{\left(1+\beta_{j} q\right)\left(2+\beta_{j} q\right)\left(3+\beta_{j} q\right)}, \\
& a_{j+1}:=1+\tilde{a}_{j} p, \quad \alpha_{j+1}:=\tilde{\alpha}_{j} p, \quad b_{j+1}:=n(p-1)+\tilde{b}_{j} p, \quad \beta_{j+1}:=3+\tilde{\beta}_{j} p, \\
& \tilde{a}_{j+1}:=a_{j} q, \quad \tilde{\alpha}_{j+1}:=1+\alpha_{j} q, \quad \tilde{b}_{j+1}:=n(q-1)+b_{j} q, \quad \tilde{\beta}_{j+1}:=3+\beta_{j} q .
\end{aligned}
$$


In the proof, it is sufficient for us to determine the sequence for $a_{j}, \tilde{a}_{j}, \alpha_{j}, \tilde{\alpha}_{j}, b_{j}$ and $\tilde{b}_{j}$ for any odd number $j \geqslant 3$. So, employing the last recursive relations with the initial value stated in (4.11), (4.12) and the formula

$$
\begin{aligned}
a_{j} & =m+a_{j-2} p q=\cdots=m\left(1+(p q)+\cdots+(p q)^{\frac{j-3}{2}}\right)+a_{1}(p q)^{\frac{j-1}{2}} \\
& =\left(a_{1}+\frac{m}{p q-1}\right)(p q)^{\frac{j-1}{2}}-\frac{m}{p q-1},
\end{aligned}
$$

one may get

$$
\begin{array}{ll}
a_{j}=\frac{p q}{p q-1}(p q)^{\frac{j-1}{2}}-\frac{1}{p q-1}, & \tilde{a}_{j}=\frac{q}{p q-1}(p q)^{\frac{j-1}{2}}-\frac{q}{p q-1}, \\
\alpha_{j}=\frac{p}{p q-1}(p q)^{\frac{j-1}{2}}-\frac{p}{p q-1}, & \tilde{\alpha}_{j}=\frac{p q}{p q-1}(p q)^{\frac{j-1}{2}}-\frac{1}{p q-1}, \\
b_{j}=\frac{(n-1) p+2 n}{2}(p q)^{\frac{j-1}{2}}-n, & \tilde{b}_{j}=\frac{(n-1) q+2 n}{2}(p q)^{\frac{j-1}{2}}-n,
\end{array}
$$

for any odd number $j$. By the same way, we deduce

$$
\begin{aligned}
& \beta_{j}=\frac{(n+2)(p q-1)+3(p+1)}{p q-1}(p q)^{\frac{j-1}{2}}-\frac{3(p+1)}{p q-1}, \\
& \tilde{\beta}_{j}=\frac{(n+2)(p q-1)+3(q+1)}{p q-1}(p q)^{\frac{j-1}{2}}-\frac{3(q+1)}{p q-1},
\end{aligned}
$$

for any odd number $j$, moreover, from the recursive relations between $\beta_{j}$ and $\tilde{\beta}_{j}$ again with even number $j$, i.e. $j-1$ is an odd number, one has

$$
\begin{aligned}
& \beta_{j}=3+\tilde{\beta}_{j-1} p=\frac{(n+2)(p q-1)+3(q+1)}{(p q-1) q}(p q)^{\frac{j}{2}}-\frac{3(p+1)}{p q-1}, \\
& \tilde{\beta}_{j}=3+\beta_{j-1} q=\frac{(n+2)(p q-1)+3(p+1)}{(p q-1) p}(p q)^{\frac{j}{2}}-\frac{3(q+1)}{p q-1} .
\end{aligned}
$$

The previous representations of $\beta_{j}, \tilde{\beta}_{j}$ imply that there exist suitable positive constants $B_{0}$ and $\widetilde{B}_{0}$, which are independent of $j$, such that $\beta_{j} \leqslant B_{0}(p q)^{\frac{j}{2}}$ and $\tilde{\beta}_{j} \leqslant \widetilde{B}_{0}(p q)^{\frac{j}{2}}$ for any $j \geqslant 1$. They immediately lead to

$$
\begin{aligned}
& D_{j} \geqslant C_{0}\left(2+\tilde{\beta}_{j-1} p\right)^{-3} \widetilde{D}_{j-1}^{p} \geqslant C_{0} \beta_{j}^{-3} \widetilde{D}_{j-1}^{p} \geqslant C_{0} B_{0}^{-3}(p q)^{-\frac{3}{2} j} \widetilde{D}_{j-1}^{p}, \\
& \widetilde{D}_{j} \geqslant \widetilde{C}_{0}\left(2+\beta_{j-1} q\right)^{-3} D_{j-1}^{q} \geqslant \widetilde{C}_{0} \tilde{\beta}_{j}^{-3} D_{j-1}^{q} \geqslant \widetilde{C}_{0} \widetilde{B}_{0}^{-3}(p q)^{-\frac{3}{2} j} D_{j-1}^{q},
\end{aligned}
$$

for any odd number $j$. Summarizing the above relations yields

$$
\begin{aligned}
& D_{j} \geqslant C_{0} \widetilde{C}_{0}^{p} B_{0}^{-3} \widetilde{B}_{0}^{-3 p}(p q)^{-\frac{3(p+1)}{2} j+\frac{3}{2} p} D_{j-2}^{p q}=: E_{0}(p q)^{-\frac{3(p+1)}{2} j} D_{j-2}^{p q}, \\
& \widetilde{D}_{j} \geqslant C_{0}^{q} \widetilde{C}_{0} B_{0}^{-3 q} \widetilde{B}_{0}^{-3}(p q)^{-\frac{3(q+1)}{2} j+\frac{3}{2} q} \widetilde{D}_{j-2}^{p q}=: \widetilde{E}_{0}(p q)^{-\frac{3(q+1)}{2} j} \widetilde{D}_{j-2}^{p q},
\end{aligned}
$$

for any odd number $j \geqslant 3$, where $E_{0}$ and $\widetilde{E}_{0}$ are suitable positive constants independent of $j$. 
Therefore, we may apply the logarithm on the recursive relationships to see

$$
\begin{aligned}
\log D_{j} \geqslant & (p q) \log D_{j-2}-\frac{3(p+1)}{2} \log (p q)+\log E_{0} \\
\geqslant & \cdots \geqslant(p q)^{\frac{j-1}{2}} \log D_{1}-\frac{3(p+1)}{2} \log (p q) \sum_{k=0}^{(j-3) / 2}\left((j-2 k)(p q)^{k}\right)+\log E_{0} \sum_{k=0}^{(j-3) / 2}(p q)^{k} \\
= & (p q)^{\frac{j-1}{2}}\left(\log D_{1}-\frac{3(p+1)(3 p q-1) \log (p q)}{2(p q-1)^{2}}+\frac{\log E_{0}}{p q-1}\right) \\
& +\frac{3(p+1)(2 p q+j(p q-1)) \log (p q)}{2(p q-1)^{2}}-\frac{\log E_{0}}{p q-1}
\end{aligned}
$$

and identically,

$$
\begin{aligned}
\log \widetilde{D}_{j} \geqslant & (p q)^{\frac{j-1}{2}}\left(\log \widetilde{D}_{1}-\frac{3(q+1)(3 p q-1) \log (p q)}{2(p q-1)^{2}}+\frac{\log \widetilde{E}_{0}}{p q-1}\right) \\
& +\frac{3(q+1)(2 p q+j(p q-1)) \log (p q)}{2(p q-1)^{2}}-\frac{\log \widetilde{E}_{0}}{p q-1},
\end{aligned}
$$

for any odd number $j \geqslant 3$, where we applied the next formula:

$$
\sum_{k=0}^{(j-3) / 2}\left((j-2 k)(p q)^{k}\right)=\frac{2+3(p q-1)}{(p q-1)^{2}}(p q)^{\frac{j-1}{2}}-\frac{2 p q+j(p q-1)}{(p q-1)^{2}} .
$$

Let us additionally consider the number $j$ fulfilling

$$
j \geqslant j_{0}:=\left\lceil\frac{2}{3 \log (p q)} \max \left\{\frac{\log E_{0}}{p+1}, \frac{\log \widetilde{E}_{0}}{q+1}\right\}-\frac{2 p q}{p q-1}\right\rceil .
$$

It means that for any odd number with $j \geqslant j_{0}$, we can estimate the multiplicative constants by

$$
\begin{aligned}
& \log D_{j} \geqslant(p q)^{\frac{j-1}{2}} \log \left(D_{1}(p q)^{-\frac{3(p+1)(3 p q-1)}{2(p q-1)^{2}}} E_{0}^{\frac{1}{q q-1}}\right)=:(p q)^{\frac{j-1}{2}} \log E_{1}, \\
& \log \widetilde{D}_{j} \geqslant(p q)^{\frac{j-1}{2}} \log \left(\widetilde{D}_{1}(p q)^{-\frac{3(q+1)(3 p q-1)}{2(p q-1)^{2}}} \widetilde{E}_{0}^{\frac{1}{p q-1}}\right)=:(p q)^{\frac{j-1}{2}} \log \widetilde{E}_{1},
\end{aligned}
$$

where $E_{1}, \widetilde{E}_{1}$ are suitable positive constants independent of $j$.

In addition, concerning $t \geqslant R$ so that $\log (R+t) \leqslant \log (2 t)$, we summarize the derived estimates and representations of constants in (4.13), (4.14) to obtain

$$
\begin{aligned}
U(t) \geqslant & \exp \left((p q)^{\frac{j-1}{2}} \log \left(E_{1} 2^{-\frac{(n-1) p}{2}-n}\left(g_{1}(t)\right)^{\frac{p q}{p q-1}}\left(g_{2}(t)\right)^{\frac{p}{p q-1}} t^{-\frac{(n-1) p}{2}+2+\frac{3(p+1)}{p q-1}}\right)\right) \\
& \times\left(g_{1}(t)\right)^{-\frac{1}{p q-1}}\left(g_{2}(t)\right)^{-\frac{p}{p q-1}}(R+t)^{n} t^{-\frac{3(p+1)}{p q-1}}
\end{aligned}
$$

and

$$
\begin{aligned}
V(t) \geqslant & \exp \left((p q)^{\frac{j-1}{2}} \log \left(\widetilde{E}_{1} 2^{-\frac{(n-1) q}{2}-n}\left(g_{1}(t)\right)^{\frac{q}{p q-1}}\left(g_{2}(t) \frac{p q}{p q-1} t^{-\frac{(n-1) q}{2}+2+\frac{3(q+1)}{p q-1}}\right)\right)\right. \\
& \times\left(g_{1}(t)\right)^{-\frac{q}{p q-1}}\left(g_{2}(t)\right)^{-\frac{1}{p q-1}}(R+t)^{n} t^{-\frac{3(q+1)}{p q-1}}
\end{aligned}
$$


for any odd number satisfying $j \geqslant j_{0}$. The assumption (2.3) is equivalent to one of the following conditions:

$$
\begin{aligned}
& \left(g_{1}(t)\right)^{\frac{p q}{p q-1}}\left(g_{2}(t)\right)^{\frac{p}{p q-1}} t^{-\frac{(n-1) p}{2}+2+\frac{3(p+1)}{p q-1}} \geqslant C(\mathscr{L}(t))^{\frac{p}{p q-1}} \\
& \left(g_{1}(t)\right)^{\frac{q}{p q-1}}\left(g_{2}(t)\right)^{\frac{p q}{p q-1}} t^{-\frac{(n-1) q}{2}+2+\frac{3(q+1)}{p q-1}} \geqslant C(\mathscr{L}(t))^{\frac{q}{p q-1}}
\end{aligned}
$$

hold. There exists a nonnegative constant $t_{1}$ such that for any $t \geqslant t_{1}$, it holds

$$
(\mathscr{L}(t))^{\frac{\min \{p, q\}}{p q-1}} \geqslant\left(\mathscr{L}\left(t_{1}\right)\right)^{\frac{\min \{p, q\}}{p q-1}}>\underbrace{C^{-1} \max \left\{E_{1}^{-1} 2^{\frac{(n-1) p}{2}+n}, \widetilde{E}_{1}^{-1} 2^{\frac{(n-1) q}{2}+n}\right\}}_{\text {independent of } j} .
$$

Therefore, for $t \geqslant \max \left\{R, t_{1}\right\}$ and letting $j \rightarrow \infty$ in (4.16) or (4.17), we may conclude that the lower bound for the functional $(U(t), V(t))$ blows up in finite time. Then, the proof of the theorem in Case 1 is complete.

\subsection{Treatment for Case 2}

Before discussing the blow-up result in Case 2, we set

$$
G_{k}(t):=\int_{0}^{t} g_{k}(\tau) \mathrm{d} \tau \text { with } G_{k}^{\prime}(t)=g_{k}(t)>0
$$

for $k=1,2$. Namely, $G_{k}(t)$ is an strictly increasing function for all $k=1,2$. Then, by the change of variable and employing integration by parts, one finds that

$$
\begin{aligned}
\int_{0}^{t} g_{k}(t-\tau) \tau^{\alpha} \mathrm{d} \tau & =\int_{0}^{t} g_{k}(\tau)(t-\tau)^{\alpha} \mathrm{d} \tau=\left.G_{k}(\tau)(t-\tau)^{\alpha}\right|_{\tau=0} ^{\tau=t}+\alpha \int_{0}^{t} G_{k}(\tau)(t-\tau)^{\alpha-1} \mathrm{~d} \tau \\
& \geqslant \begin{cases}G_{k}\left(t_{0}\right) & \text { if } \alpha=0, \\
\alpha \int_{t_{0}}^{t} G_{k}(\tau)(t-\tau)^{\alpha-1} \mathrm{~d} \tau & \text { if } \alpha>0,\end{cases} \\
& \geqslant G_{k}\left(t_{0}\right)\left(t-t_{0}\right)^{\alpha}
\end{aligned}
$$

for any $t \geqslant t_{0}$, where $\alpha \geqslant 0$ and we used $G_{k}(0)=0$ and monotonous properties of the function $G_{k}(t)$ for all $k=1,2$. Here, we shrank the domain of the integration from $[0, t]$ into $\left[t_{0}, t\right]$. Hence, the treatment (4.18) motivate us to deal with the fast decay memory kernel in a better way.

We now can deduce the first lower bound estimates from (4.9) that

$$
\begin{aligned}
U(t) & \geqslant C_{1} \widetilde{C}_{2}^{p} \int_{0}^{t} \int_{0}^{\eta} \int_{0}^{s} g_{1}(s-\tau)(R+\tau)^{-\frac{n-1}{2} p} \tau^{n-1} \mathrm{~d} \tau \mathrm{d} s \mathrm{~d} \eta \\
& \geqslant C_{1} \widetilde{C}_{2}^{p} G_{1}\left(t_{0}\right)(R+t)^{-\frac{n-1}{2} p} \int_{t_{0}}^{t} \int_{t_{0}}^{\eta}\left(s-t_{0}\right)^{n-1} \mathrm{~d} s \mathrm{~d} \eta \\
& \geqslant \frac{C_{1} \widetilde{C}_{2}^{p}}{n(n+1)} G_{1}\left(t_{0}\right)(R+t)^{-\frac{n-1}{2} p}\left(t-t_{0}\right)^{n+1}
\end{aligned}
$$

for any $t \geqslant t_{0}$, where in the first line of the chain inequality, the derived estimate (4.18) was applied. Analogously, the estimate (4.10) shows

$$
V(t) \geqslant \frac{\widetilde{C}_{1} C_{2}^{q}}{n(n+1)} G_{2}\left(t_{0}\right)(R+t)^{-\frac{n-1}{2} q}\left(t-t_{0}\right)^{n+1}
$$


for any $t \geqslant t_{0}$. That is to say that we have derived the estimates for the functionals from the below as follows:

$$
\begin{array}{ll}
U(t) \geqslant Q_{1}(R+t)^{-\theta_{1}}\left(t-L_{1} t_{0}\right)^{\sigma_{1}} & \text { for any } t \geqslant L_{1} t_{0}, \\
V(t) \geqslant \widetilde{Q}_{1}(R+t)^{-\tilde{\theta}_{1}}\left(t-L_{1} t_{0}\right)^{\tilde{\sigma}_{1}} & \text { for any } t \geqslant L_{1} t_{0},
\end{array}
$$

where we set $L_{1}:=1$ and the constants are given by

$$
\begin{aligned}
& Q_{1}:=\frac{C_{1} \widetilde{C}_{2}^{p}}{n(n+1)} G_{1}\left(t_{0}\right), \quad \widetilde{Q}_{1}:=\frac{\widetilde{C}_{1} C_{2}^{q}}{n(n+1)} G_{2}\left(t_{0}\right), \\
& \theta_{1}:=\frac{n-1}{2} p, \quad \sigma_{1}:=n+1, \quad \tilde{\theta}_{1}:=\frac{n-1}{2} q, \quad \tilde{\sigma}_{1}:=n+1 .
\end{aligned}
$$

Similarly to the previous subsection, we will demonstrate the sequence of lower bounds for $U(t)$ and $V(t)$ by the next way:

$$
\begin{array}{ll}
U(t) \geqslant Q_{j}(R+t)^{-\theta_{j}}\left(t-L_{j} t_{0}\right)^{\sigma_{j}} & \text { for any } t \geqslant L_{j} t_{0}, \\
V(t) \geqslant \widetilde{Q}_{j}(R+t)^{-\tilde{\theta}_{j}}\left(t-L_{j} t_{0}\right)^{\tilde{\sigma}_{j}} & \text { for any } t \geqslant L_{j} t_{0},
\end{array}
$$

where $\left\{Q_{j}\right\}_{j \geqslant 1},\left\{\widetilde{Q}_{j}\right\}_{j \geqslant 1},\left\{\theta_{j}\right\}_{j \geqslant 1},\left\{\tilde{\theta}_{j}\right\}_{j \geqslant 1},\{\sigma\}_{j \geqslant 1}$ and $\{\tilde{\sigma}\}_{j \geqslant 1}$ are sequences of nonnegative real numbers that will be determined later. Furthermore, motivated by the recent paper [3], we construct $\left\{L_{j}\right\}_{j \geqslant 1}$ to be the sequence of the partial products of the convergent infinite product

$$
\prod_{k=1}^{\infty} \ell_{k} \text { with } \ell_{k}:=1+(p q)^{-\frac{k-1}{2}} \text { for any } k \geqslant 1,
$$

that is,

$$
L_{j}:=\prod_{k=1}^{j} \ell_{k} \text { for any } j \geqslant 1 .
$$

Clearly, the convergent property can be easily obtained from the ratio test method and the fact that $\lim _{k \rightarrow \infty}\left(\ln \ell_{k+1}\right) /\left(\ln \ell_{k}\right)=(p q)^{-1 / 2}<1$. Note that the initial value of the previous sequences was stated in (4.19) as well as (4.20).

Let us begin with processing the inductive step by combining (4.21), (4.22) and (4.4), (4.5). We assume (4.21) and (4.22) hold for $j$, and our goal is to prove them also hold for $j+1$. By this way, one may get

$$
U(t) \geqslant C_{0} \widetilde{Q}_{j}^{p}(R+t)^{-n(p-1)-\tilde{\theta}_{j} p} \int_{L_{j} t_{0}}^{t} \int_{L_{j} t_{0}}^{\eta} \int_{L_{j} t_{0}}^{s} g_{1}(s-\tau)\left(\tau-L_{j} t_{0}\right)^{\tilde{\sigma}_{j} p} \mathrm{~d} \tau \mathrm{d} s \mathrm{~d} \eta .
$$

Let us discuss the estimate the $\tau$-integral in the previous line. By changing of the variable and using integration by parts associated with $G_{1}(0)=0$, we have

$$
\begin{aligned}
\int_{L_{j} t_{0}}^{s} g_{1}(s-\tau)\left(\tau-L_{j} t_{0}\right)^{\tilde{\sigma}_{j} p} \mathrm{~d} \tau & =\int_{0}^{s-L_{j} t_{0}} g_{1}(\tau)\left(s-L_{j} t_{0}-\tau\right)^{\tilde{\sigma}_{j} p} \mathrm{~d} \tau \\
& =\tilde{\sigma}_{j} p \int_{0}^{s-L_{j} t_{0}} G_{1}(\tau)\left(s-L_{j} t_{0}-\tau\right)^{\tilde{\sigma}_{j} p-1} \mathrm{~d} \tau \\
& \geqslant \tilde{\sigma}_{j} p \int_{L_{j} t_{0}\left(\ell_{j+1}-1\right)}^{s-L_{j} t_{0}} G_{1}(\tau)\left(s-L_{j} t_{0}-\tau\right)^{\tilde{\sigma}_{j} p-1} \mathrm{~d} \tau \\
& \geqslant G_{1}\left(L_{j} t_{0}\left(\ell_{j+1}-1\right)\right)\left(s-L_{j} t_{0} \ell_{j+1}\right)^{\tilde{\sigma}_{j} p}
\end{aligned}
$$


for any $s \geqslant L_{j+1} t_{0}$, where we shrank the domain by using the fact that

$$
s \geqslant L_{j+1} t_{0}=L_{j} t_{0} \ell_{j+1} \text { implies } s-L_{j} t_{0} \geqslant L_{j} t_{0}\left(\ell_{j+1}-1\right)>0 .
$$

Let us estimate $G_{1}\left(L_{j} t_{0}\left(\ell_{j+1}-1\right)\right)$ from below now by introducing

$$
L:=\lim _{j \rightarrow \infty} L_{j}=\prod_{j=1}^{\infty} \ell_{j}=\prod_{j=1}^{\infty}\left(1+(p q)^{-\frac{j-1}{2}}\right)>1 .
$$

Since $\ell_{j}>1$ the sequence $\left\{L_{j}\right\}_{j \geqslant 1}$ is converging to $L$ as $j \rightarrow \infty$. So, we may claim $1 \leqslant L_{j} \leqslant L$ for all $j \geqslant 1$. There is a positive real integer $j_{m}$ such that if $j \geqslant j_{m}$, then it holds

$$
0<L_{j} t_{0}\left(\ell_{j+1}-1\right) \leqslant L t_{0}(p q)^{-j / 2} \ll 1 .
$$

At this time, according to our assumptions $g_{1}(t) \in \mathscr{C}^{2}([0, T])$ and $g_{1}^{\prime \prime}(0)>0$, it would lead to

$$
\begin{aligned}
G_{1}\left(L_{j} t_{0}\left(\ell_{j+1}-1\right)\right) & \geqslant G_{1}(0)+g_{1}(0) L_{j} t_{0}(p q)^{-\frac{j}{2}}+\frac{g_{1}^{\prime}(0)}{2} L_{j}^{2} t_{0}^{2}(p q)^{-j}+g_{1}^{\prime \prime}(0) \odot\left((p q)^{-\frac{3}{2} j} L_{j}^{3}\right) \\
& \geqslant(p q)^{-j} L_{j} t_{0}\left((p q)^{\frac{j}{2}} g_{1}(0)+\frac{g_{1}^{\prime}(0)}{2} L_{j} t_{0}\right)+g_{1}^{\prime \prime}(0) \odot\left((p q)^{-\frac{3}{2} j}\right) \\
& \geqslant(p q)^{-j} t_{0}\left((p q)^{\frac{j}{2}} g_{1}(0)-\frac{-g_{1}^{\prime}(0)}{2} L t_{0}\right) \\
& \geqslant C_{3}(p q)^{-j}
\end{aligned}
$$

for any $j \geqslant \max \left\{j_{m}, j_{1}\right\}$, where $C_{3}>0$ and we denoted

$$
j_{1}:= \begin{cases}1 & \text { if } g_{1}^{\prime}(0)>0 \\ \left\lceil 2 \log _{p q}\left(\frac{1}{g_{1}(0)}-\frac{g_{1}^{\prime}(0) L t_{0}}{2 g_{1}(0)}\right)\right\rceil & \text { if } g_{1}^{\prime}(0) \leqslant 0\end{cases}
$$

We should emphasize that the definition of $j_{1}$ when $g_{1}^{\prime}(0) \leqslant 0$ turn out from

$$
(p q)^{\frac{j}{2}} g_{1}(0)-\frac{-g_{1}^{\prime}(0)}{2} L t_{0} \geqslant 1
$$

for any integer $j \geqslant j_{0}$

Summing up the derived estimates, we conclude

$$
\begin{aligned}
U(t) & \geqslant C_{0} C_{3}(p q)^{-j} \widetilde{Q}_{j}^{p}(R+t)^{-n(p-1)-\tilde{\theta}_{j} p} \int_{L_{j+1} t_{0}}^{t} \int_{L_{j+1} t_{0}}^{\eta}\left(s-L_{j} t_{0} \ell_{j+1}\right)^{\tilde{\sigma}_{j} p} \mathrm{~d} s \mathrm{~d} \eta \\
& \geqslant \frac{C_{0} C_{3}(p q)^{-j} \widetilde{Q}_{j}^{p}}{\left(\tilde{\sigma}_{j} p+1\right)\left(\tilde{\sigma}_{j} p+2\right)}(R+t)^{-n(p-1)-\tilde{\theta}_{j} p}\left(t-L_{j+1} t_{0}\right)^{\tilde{\sigma}_{j} p+2}
\end{aligned}
$$

for any $t \geqslant L_{j+1} t_{0}$ and $j \geqslant \max \left\{j_{m}, j_{1}\right\}$.

By the same way of deduction as the lower bound estimates for $U(t)$, there exists a positive constant $\widetilde{C}_{3}$ such that

$$
V(t) \geqslant \frac{\widetilde{C}_{0} \widetilde{C}_{3}(p q)^{-j} Q_{j}^{q}}{\left(\sigma_{j} q+1\right)\left(\sigma_{j} q+2\right)}(R+t)^{-n(q-1)-\theta_{j} q}\left(t-L_{j+1} t_{0}\right)^{\sigma_{j} q+2}
$$


for any $t \geqslant L_{j+1} t_{0}$ and $j \geqslant \max \left\{j_{m}, \tilde{j}_{1}\right\}$ with

$$
\tilde{j}_{1}:=\left\{\begin{array}{lll}
1 & \text { if } g_{2}^{\prime}(0)>0 \\
\left\lceil 2 \log _{p q}\left(\frac{1}{g_{2}(0)}-\frac{g_{2}^{\prime}(0) L t_{0}}{2 g_{2}(0)}\right)\right\rceil & \text { if } g_{2}^{\prime}(0) \leqslant 0 .
\end{array}\right.
$$

In other words, the desired lower bound estimates (4.21) and (4.22) are valid for $j+1$ if

$$
\begin{aligned}
Q_{j+1} & :=\frac{C_{0} C_{3}(p q)^{-j} \widetilde{Q}_{j}^{p}}{\left(\tilde{\sigma}_{j} p+1\right)\left(\tilde{\sigma}_{j} p+2\right)}, \quad \widetilde{Q}_{j+1}:=\frac{\widetilde{C}_{0} \widetilde{C}_{3}(p q)^{-j} Q_{j}^{q}}{\left(\sigma_{j} q+1\right)\left(\sigma_{j} q+2\right)}, \\
\theta_{j+1} & :=n(p-1)+\tilde{\theta}_{j} p, \quad \sigma_{j+1}:=\tilde{\sigma}_{j} p+2, \quad \tilde{\theta}_{j+1}:=n(q-1)+\theta_{j} q, \quad \tilde{\sigma}_{j+1}:=\sigma_{j} q+2 .
\end{aligned}
$$

Similarly to the treatment in the last subsection, for any odd integer $j$ such that $j \geqslant \max \left\{j_{m}, j_{1}, \tilde{j}_{1}\right\}$, we employ iteratively the obtained relations to get

$$
\theta_{j}=\frac{2 n+(n-1) p}{2}(p q)^{\frac{j-1}{2}}-n, \quad \tilde{\theta}_{j}=\frac{2 n+(n-1) q}{2}(p q)^{\frac{j-1}{2}}-n .
$$

What's more, we obtain

$$
\begin{aligned}
& \sigma_{j}=\frac{(n+1)(p q-1)+2(p+1)}{p q-1}(p q)^{\frac{j-1}{2}}-\frac{2(p+1)}{p q-1}, \\
& \tilde{\sigma}_{j}=\frac{(n+1)(p q-1)+2(q+1)}{p q-1}(p q)^{\frac{j-1}{2}}-\frac{2(q+1)}{p q-1},
\end{aligned}
$$

for any odd number $j \geqslant \max \left\{j_{m}, j_{1}, \tilde{j}_{1}\right\}$, and

$$
\begin{aligned}
& \sigma_{j}=\tilde{\sigma}_{j-1} p+2=\frac{(n+1)(p q-1)+2(q+1)}{(p q-1) q}(p q)^{\frac{j}{2}}-\frac{2(p+1)}{p q-1}, \\
& \tilde{\sigma}_{j}=\sigma_{j-1} q+2=\frac{(n+1)(p q-1)+2(q+1)}{(p q-1) p}(p q)^{\frac{j}{2}}-\frac{2(q+1)}{p q-1},
\end{aligned}
$$

for any even number $j \geqslant \max \left\{j_{m}, j_{1}, \tilde{j}_{1}\right\}$. It immediately leads to $\sigma_{j} \leqslant B_{1}(p q)^{\frac{j}{2}}$ and $\tilde{\sigma}_{j} \leqslant \widetilde{B}_{1}(p q)^{\frac{j}{2}}$ with suitable positive constants $B_{1}$ and $\widetilde{B}_{1}$ independent of $j$.

Next, by using the relation between $Q_{j}$ and $\widetilde{Q}_{j}$ such that

$$
\begin{aligned}
& Q_{j} \geqslant \frac{C_{0} C_{3}}{B_{1}^{2}}(p q)^{-2 j+1} \widetilde{Q}_{j-1}^{p} \geqslant \frac{C_{0} \widetilde{C}_{0}^{p} C_{3} \widetilde{C}_{3}^{p}(p q)^{3 p+1}}{B_{1}^{2} \widetilde{B}_{1}^{2 p}}(p q)^{-2 j(p+1)} Q_{j-2}^{p q}=: E_{2}(p q)^{-2 j(p+1)} Q_{j-2}^{p q}, \\
& \widetilde{Q}_{j} \geqslant \frac{\widetilde{C}_{0} \widetilde{C}_{3}}{\widetilde{B}_{1}^{2}}(p q)^{-2 j+1} Q_{j-1}^{q} \geqslant \frac{\widetilde{C}_{0} C_{0}^{q} C_{3}^{q} \widetilde{C}_{3}(p q)^{3 q+1}}{B_{1}^{2 q} \widetilde{B}_{1}^{2}}(p q)^{-2 j(q+1)} \widetilde{Q}_{j-2}^{p q}=: \widetilde{E}_{2}(p q)^{-2 j(q+1)} \widetilde{Q}_{j-2}^{p q},
\end{aligned}
$$

where $E_{2}$ and $\widetilde{E}_{2}$ are suitable positive constants, one may obtain by applying the logarithmic function on the both sides of them to have

$$
\begin{aligned}
\log Q_{j} \geqslant & (p q)^{\frac{j-1}{2}} \log Q_{1}-2(p+1) \log (p q) \sum_{k=0}^{(j-3) / 2}\left((j-2 k)(p q)^{k}\right)+\log E_{2} \sum_{k=0}^{(j-3) / 2}(p q)^{k} \\
= & (p q)^{\frac{j-1}{2}}\left(\log Q_{1}-\frac{2(p+1)(3 p q-1) \log (p q)}{(p q-1)^{2}}+\frac{\log E_{2}}{p q-1}\right) \\
& +\frac{2(p+1)(2 p q+j(p q-1)) \log (p q)}{(p q-1)^{2}}-\frac{\log E_{2}}{p q-1}
\end{aligned}
$$


as well as

$$
\begin{aligned}
\log \widetilde{Q}_{j} \geqslant & (p q)^{\frac{j-1}{2}} \log \widetilde{Q}_{1}-\frac{2(q+1)}{2} \log (p q) \sum_{k=0}^{(j-3) / 2}\left((j-2 k)(p q)^{k}\right)+\log \widetilde{E}_{2} \sum_{k=0}^{(j-3) / 2}(p q)^{k} \\
= & (p q)^{\frac{j-1}{2}}\left(\log \widetilde{Q}_{1}-\frac{2(q+1)(3 p q-1) \log (p q)}{(p q-1)^{2}}+\frac{\log \widetilde{E}_{2}}{p q-1}\right) \\
& +\frac{2(q+1)(2 p q+j(p q-1)) \log (p q)}{(p q-1)^{2}}-\frac{\log \widetilde{E}_{2}}{p q-1}
\end{aligned}
$$

for any odd number $j \geqslant \max \left\{j_{m}, j_{1}, \tilde{j}_{1}\right\}$, where we used (4.15) again. Fixing

$$
j_{2}:=\left\lceil\frac{1}{2 \log (p q)} \max \left\{\frac{\log E_{2}}{p+1}, \frac{\log \widetilde{E}_{2}}{q+1}\right\}-\frac{2 p q}{p q-1}\right\rceil,
$$

therefore, for any odd number $j \geqslant \max \left\{j_{m}, j_{1}, \tilde{j}_{1}, j_{2}\right\}$, we can estimate the multiplicative constants as follows:

$$
\begin{aligned}
& \log Q_{j} \geqslant(p q)^{\frac{j-1}{2}} \log \left(Q_{1}(p q)^{-\frac{2(p+1)(3 p q-1)}{(p q-1)^{2}}} E_{2}^{\frac{1}{p q-1}}\right)=:(p q)^{\frac{j-1}{2}} \log E_{3}, \\
& \log \widetilde{Q}_{j} \geqslant(p q)^{\frac{j-1}{2}} \log \left(\widetilde{Q}_{1}(p q)^{-\frac{2(q+1)(3 p q-1)}{(p q-1)^{2}}} \widetilde{E}_{2}^{\frac{1}{p q-1}}\right)=:(p q)^{\frac{j-1}{2}} \log \widetilde{E}_{3},
\end{aligned}
$$

where $E_{3}, \widetilde{E}_{3}$ are suitable positive constants independent of $j$.

Let us recall the limit (4.25), which leads to (4.21) and (4.22) for $t \geqslant L t_{0}$. Concerning $t \geqslant$ $\max \left\{R, 2 L t_{0}\right\}$ showing

$$
\log (R+t) \leqslant \log (2 t) \text { and } \log \left(t-L t_{0}\right) \geqslant \log (t / 2)
$$

we summarize the derived estimates and representations of constants in (4.21), (4.22) to obtain

$$
\begin{aligned}
& U(t) \geqslant \exp \left((p q)^{\frac{j-1}{2}} \log \left(Q_{1} 2^{-\frac{2 n+(n-1) p}{2}-\frac{(n+1)(p q-1)+2(p+1)}{p q-1}} t^{-\frac{(n-1) p}{2}+1+\frac{2(p+1)}{p q-1}}\right)\right)(R+t)^{n} t^{-\frac{2(p+1)}{p q-1}}, \\
& V(t) \geqslant \exp \left((p q)^{\frac{j-1}{2}} \log \left(\widetilde{Q}_{1} 2^{-\frac{2 n+(n-1) q}{2}}-\frac{(n+1)(p q-1)+2(q+1)}{p q-1} t^{-\frac{(n-1) q}{2}+1+\frac{2(q+1)}{p q-1}}\right)\right)(R+t)^{n} t^{-\frac{2(q+1)}{p q-1}},
\end{aligned}
$$

for any odd number fulfilling $j \geqslant \max \left\{j_{m}, j_{1}, \tilde{j}_{1}, j_{2}\right\}$.

Let us recall the condition (2.4) which is equivalent to

$$
-\frac{(n-1) p}{2}+1+\frac{2(p+1)}{p q-1}>0 \text { or }-\frac{(n-1) q}{2}+1+\frac{2(q+1)}{p q-1}>0 .
$$

The power of $t$ in the exponential function is positive. Eventually, by taking $t \geqslant\left\{R, 2 L t_{0}\right\}$ and

$$
t>\min \left\{\left(Q_{1} 2^{2 n+1+\frac{n-1}{2} p+\frac{2(p+1)}{p q-1}}\right)^{-\frac{(n-1) p}{2}+1+\frac{2(p+1)}{p q-1}},\left(\widetilde{Q}_{1} 2^{2 n+1+\frac{n-1}{2} q+\frac{2(q+1)}{p q-1}}\right)^{-\frac{(n-1) q}{2}+1+\frac{2(q+1)}{p q-1}}\right\},
$$

we may immediately find blow-up for the lower bounds of the functional $(U(t), V(t))$ in $(4.26)$ and (4.27) as $j \rightarrow \infty$. In conclusion, this concludes the proof in Case 2. 


\section{$5 \quad$ Final remarks}

Throughout this paper, we have mainly derived blow-up of energy solutions for the weakly coupled systems (1.1) for distinct memory terms in general forms. Precisely, we determine a threshold $t^{-1}$ for the blow-up condition. However, it is still open to determine the critical condition or the critical curve for (1.1) under certain assumptions on memory kernels.

Let us now show some open problems strongly related to the topic in this paper and give some conjectures on them. In the following discussion, we generally assume $g_{k}(t) \in L^{1}([0, T])$ with $T \leqslant \infty$ for $k=1,2$.

- If $g_{k}(t) \gtrsim t^{-1}$ for any $t \geqslant t_{0}$ with $t_{0} \in[0, T)$ and $g_{k}^{\prime}(t) \leqslant 0$ for $k=1,2$, then it is still open for the existence of global (in time) solution in the supercritical case

$$
g_{1}(t) g_{2}(t) \max \left\{\left(g_{1}(t)\right)^{q-1} t^{2 q+1 / p},\left(g_{2}(t)\right)^{p-1} t^{2 p+1 / q}\right\} \lesssim t^{\frac{n-1}{2}(p q-1)-3}
$$

and nonexistence of local (in time) solution in the critical case

$$
g_{1}(t) g_{2}(t) \max \left\{\left(g_{1}(t)\right)^{q-1} t^{2 q+1 / p},\left(g_{2}(t)\right)^{p-1} t^{2 p+1 / q}\right\} \asymp t^{\frac{n-1}{2}(p q-1)-3} .
$$

We should underline that the conjecture in the critical case for $p=q$ and $g_{1}(t)=g_{2}(t)=t^{-\gamma}$ with $\gamma \in(0,1)$, from point of view of the single semilinear equation, is true and we refer to Theorem 2 in [2]. Hence, the conjectures seem reasonable.

- If $g_{k}(t) \lesssim t^{-1}$ for any $t \geqslant t_{0}$ with $t_{0} \in[0, T)$ and $g_{k}(t) \in \mathscr{C}^{2}([0, T])$ with $g_{k}^{\prime \prime}(0)>0$ for $k=1,2$, then it is still open for the existence of global (in time) solution in the supercritical case

$$
\max \left\{\frac{p+2+q^{-1}}{p q-1}, \frac{q+2+p^{-1}}{p q-1}\right\}<\frac{n-1}{2},
$$

and nonexistence of local (in time) solution in the critical case

$$
\max \left\{\frac{p+2+q^{-1}}{p q-1}, \frac{q+2+p^{-1}}{p q-1}\right\}=\frac{n-1}{2} .
$$

We expect the nonexistence in the critical case can be proved by constructing a weighted space average as the functional, whose dynamic can be studied in the iteration procedure $[1,3]$. By this way, the conjectures seem reasonable too.

\section{Acknowledgments}

The Ph.D. study of Wenhui Chen are supported by Sächsisches Landesgraduiertenstipendium. This work was partially written while Wenhui Chen was a Ph.D. student at TU Freiberg. The author thanks Michael Reissig (TU Bergakademie Freiberg), Alessandro Palmieri (University of Pisa) and Giovanni Girardi (University of Bari) for the suggestions in the preparation of the paper. 


\section{References}

[1] R. Agemi, Y. Kurokawa, H. Takamura, Critical curve for $p$ - $q$ systems of nonlinear wave equations in three space dimensions, J. Differential Equations 167 (2000) 87-133.

[2] W. Chen, A. Palmieri, Blow-up result for a semilinear wave equation with a nonlinear memory term, INdAM: Anomalies in PDEs (in press) (2020) 20 pages.

[3] W. Chen, A. Palmieri, Nonexistence of global solutions for the semilinear Moore-GibsonThompson equation in the conservative case, Discrete Contin. Dyn. Syst. (in press) (2020) 28 pages.

[4] M. D'Abbicco, A wave equation with structural damping and nonlinear memory, NoDEA Nonlinear Differential Equations Appl. 21 (2014) 751-773.

[5] M. D'Abbicco, The influence of a nonlinear memory on the damped wave equation, Nonlinear Anal. 95 (2014) 130-145.

[6] I. Dannawi, M. Kirane, A.Z. Fino, Finite time blow-up for damped wave equations with spacetime dependent potential and nonlinear memory, NoDEA Nonlinear Differential Equations Appl. 25 (2018) 38.

[7] T.A. Dao, A.Z. Fino, Blow up results for semi-linear structural damped wave model with nonlinear memory, preprint (2020). arxiv.org/abs/2002.06582

[8] D. Del Santo, Global existence and blow-up for a hyperbolic system in three space dimensions, Rend. Istit. Mat. Univ. Trieste 29 (1997) 115-140.

[9] D. Del Santo, E. Mitidieri, Blow-up of solutions of a hyperbolic system: The critical case, Differential Equations 34 (1998) 1167-1163.

[10] D. Del Santo, V. Georgiev, E. Mitidieri, Global existence of the solutions and formation of singularities for a class of hyperbolic system, in F. Colombini, N. Lerner (Eds.) Geometrical Optics and Related Topics. Progress in Nonlinear Differential Equations and Their Applications, vol 32. Birkhäuser, Boston, MA, (1997).

[11] M.R. Ebert, M. Reissig, Methods for Partial Differential Equations, Birkhäuser Basel, Germany, (2018).

[12] A.Z. Fino, Critical exponent for damped wave equations with nonlinear memory, Nonlinear Anal. 74 (2011) 5495-5505.

[13] V. Georgiev, H. Lindblad, C.D. Sogge, Weighted Strichartz estimates and global existence for semilinear wave equations, Amer. J. Math. 119 (1997) 1291-1319.

[14] V. Georgiev, H. Takamura, Y. Zhou, The lifespan of solutions to nonlinear systems of a high-dimensional wave equation, Nonlinear Anal. 64 (2006) 2215-2250.

[15] R.T. Glassey, Existence in the large for $\square u=F(u)$ in two space dimensions, Math. Z. 178 (1981) 233-261. 
[16] R.T. Glassey, Finite-time blow-up for solutions of nonlinear wave equations, Math. Z. 177 (1981) 323-340.

[17] H. Jiao, Z. Zhou, An elementary proof of the blow-up for semilinear wave equation in high space dimensions, J. Differential Equations 189 (2003) 355-365.

[18] F. John, Blow-up of solutions of nonlinear wave equations in three space dimensions, Manuscripta Math. 28 (1979) 235-268.

[19] T. Kato, Blow-up of solutions of some nonlinear hyperbolic equations, Comm. Pure Appl. Math. 33 (1980) 501-505.

[20] Y. Kurokawa, The lifespan of radially symmetric solutions to nonlinear systems of odd dimensional wave equations, Tsukuba J. Math. 60 (2005) 1239-1275.

[21] Y. Kurokawa, H. Takamura, A weighted pointwise estimate for two dimensional wave equations and its applications to nonlinear systems, Tsukuba J. Math. 27 (2003) 417-448.

[22] Y. Kurokawa, H. Takamura, K. Wakasa, The blow-up and lifespan of solutions to systems of semilinear wave equation with critical exponents in high dimensions, Differential Integral Equations 25 (2012) 363-382.

[23] N.A. Lai, J. Liu, J. Zhao, Blow up for initial-boundary value problem of wave equation with a nonlinear memory in 1-D, Chin. Ann. Math. Ser. B 38 (2017) 827-838.

[24] N.A. Lai, H. Takamura, Nonexistence of global solutions of nonlinear wave equations with weak time-dependent damping related to Glassey's conjecture, Differential Integral Equations 32 (2019) 37-48.

[25] N.A. Lai, H. Takamura, K. Wakasa, Blow-up for semilinear wave equations with the scale invariant damping and super-Fujita exponent, J. Differential Equations 263 (2017) 5377-5394.

[26] H. Lindblad, C.D. Sogge, Long-time existence for small amplitude semilinear wave equations, Amer. J. Math. 118 (1996) 1047-1135.

[27] A. Palmieri, H. Takamura, Blow-up for a weakly coupled system of semilinear damped wave equations in the scattering case with power nonlinearities, Nonlinear Anal. 187 (2019) 467-492.

[28] M. Pellicer, B. Said-Houari, Wellposedness and decay rates for the Cauchy problem of the Moore-Gibson-Thompson equation arising in high intensity ultrasound, Appl. Math. Optim. 80 (2019) 447-478.

[29] B. Said-Houari, M. Kirane, Global nonexistence results for a class of hyperbolic systems, Nonlinear Anal. 74 (2011) 6130-6143.

[30] J. Schaeffer, The equation $u_{t t}-\Delta u=|u|^{p}$ for the critical value of $p$, Proc. Roy. Soc. Edinburgh Sect. A. 101 (1985) 31-44.

[31] T.C. Sideris, Nonexistence of global solutions to semilinear wave equations in high dimensions, J. Differential Equations 52 (1984) 378-406. 
[32] W.A. Strauss, Nonlinear scattering theory at low energy, J. Funct. Anal. 41 (1981) 110-133.

[33] D. Tataru, Strichartz estimates in the hyperbolic space and global existence for the semilinear wave equation, Trans. Amer. Math. Soc. 353 (2001) 795-807.

[34] B.T. Yordanov, Q.S. Zhang, Finite time blow up for critical wave equations in high dimensions, J. Funct. Anal. 231 (2006) 361-374.

[35] Y. Zhou, Cauchy problem for semilinear wave equations in four space dimensions with small initial data, J. Partial Differential Equations 8 (1995) 135-144.

[36] Y. Zhou, Blow up of solutions to semilinear wave equations with critical exponent in high dimensions, Chin. Ann. Math. Ser. B 28 (2007) 205-212. 